\title{
Simulation of supercritical fuel injection with condensation
}

\author{
Lu Qiu ${ }^{1, a)}$ and Rolf D. Reitz ${ }^{1, b)}$
}

\begin{abstract}
Supercritical injection and the accompanying potential condensation processes were numerically investigated with a consistent treatment for both the fluid dynamics and thermodynamics with a realistic equation of state model. Qualitative and quantitative improvement was seen on the predicted injectant density with real gas simulations when compared to available experimental data, emphasizing the importance of thermodynamic property non-ideality at supercritical conditions. Recently developed phase stability and equilibrium solvers utilizing fundamental thermodynamics principles were also applied to capture phase transitions. To our knowledge, this is the first comprehensive simulation against experimental data of supercritical injection with phase separation. Furthermore, the simulation results were found to agree with the experimental results in three aspects related to the phase change. The first observation is that condensation is predicted to occur if and only if the temperature difference between the injectant and ambient is large enough to promote strong heat transfer interactions. The simulations also show that condensation becomes intensified when the chamber temperature is further reduced. Second, such condensation is only possible for supercritical-to-subcritical but not for supercritical-to-supercritical injections. Third, a condensed liquid phase is found to form at the jet boundary where the energy and mixing interactions between the "hot" injectant and the "cold" surrounding gas are strong. The intensive local heat exchange finally sends the mixture into the two-phase region by crossing the dew point line with condensation.
\end{abstract}

Key words: Supercritical injection; Phase equilibrium; Phase stability; Compressible two-phase flows; Equation of state; Isoenergetic-isochoric flash 


\section{Introduction}

Liquid fuel injection is a common process that occurs in a number of energy conversion devices, such as gas turbines, diesel engines and gasoline direct-injection (GDI) engines. Understanding the complex physical processes (e.g., liquid jet breakup, atomization, turbulent mixing, evaporation, etc.) is critical to better utilize the fossil fuels because the fuel-air mixing plays a critical role in the subsequent gas phase combustion processes. A number of classical physical breakup and atomization models have been widely accepted and used in spray simulations. For instance, the Kelvin-Helmholtz Rayleigh-Taylor breakup model [1-3], which identifies the fastest growing wave (with highest surface instability) on a droplet surface and considers it to be responsible for the subsequent breakup, has been widely used in state-of-the-art multidimensional diesel spray simulations [4-10]. Current diesel engines are generally equipped with high pressure injectors, and to enhance the fuel/air mixing processes, the injection pressure has been increased from around 1000 bar to as high as 2000 to 3000 bar in recent years. Under such high pressure conditions, the injected fuel is far above its thermodynamic critical pressure so the fuel is in a highly compressed liquid state.

Recent large eddy simulation (LES) modeling shows that, under high injection pressure conditions, an n-heptane fuel jet may be approximated as a dense fluid, for which the traditional atomization and breakup processes do not exist [11]. Dahms and Oefelein [12] applied a linear gradient theory to quantify at what operating pressures the phase transition processes can be analyzed using classical two-phase atomization theories, or quite differently, based on diffusion-dominated mixing. Some potential benefits of injecting supercritical diesel fuels in engines have also been experimentally proved. Anitescu et al. [13] found that the jet spreading angle was enlarged and the observable jet core length was reduced, which is direct evidence that fuel/air mixing was significantly enhanced when dieseline (a mixture of diesel and gasoline) was injected in a supercritical state. It was also argued that such distinctive behavior could further reduce the fuel impingement and wall film formation in direct injection engines [13]. This is especially beneficial to advanced low temperature combustion (LTC) strategies for which early fuel injection is generally a prerequisite to improve the homogeneity of the fuel air mixture. For instance, in dual-fuel reactivity controlled compression ignition (RCCI) combustion, the first injection is as early as 58 crank angles before top dead center [14], which can introduce a significant amount of fuel impingement on the cylinder walls and in the piston-liner region. 
Fluid behavior under supercritical conditions has been widely investigated and has been shown to be drastically different from that under subcritical conditions. Newman and Brzustowski [15] reported the first experimental work on supercritical carbon dioxide $\left(\mathrm{CO}_{2}\right)$ as the injectant and nitrogen $\left(\mathrm{N}_{2}\right)$ as the ambient, for which the pressure was above the critical pressure of $\mathrm{CO}_{2}$. Experiments show that the higher the ambient gas density at high temperature, the finer the spray (e.g., reduced droplet size and number density), similar to the situation for subcritical environments. In the following, otherwise specified, supercritical or subcritical is used with reference to the critical properties of the injectant. In a supercritical temperature environment, liquid droplets can reach their critical temperature during the heating process, resulting in a significant reduction of surface tension.

Analysis at supercritical temperatures using turbulent submerged single-phase jet theory (e.g., velocity and temperature similarities) in Ref. [15] showed that the predicted jet flow behavior (e.g., variation of the axial mean velocity, location of jet boundaries) resembled jets at supercritical chamber temperatures. When the local evaporating rate was low due to high concentrations of the injectant, and when the injection velocity was high, the authors concluded that the "spray" could be characterized as "single-phase" turbulent gas jet. A thorough review is beyond the scope of the present paper, but can be found in a recent review article by Chehroudi [16], which includes many well-illustrating experimental images. The major conclusion regarding a single jet issuing into a quiescent chamber is that: when the chamber pressure exceeds the critical pressure of the injectant, classical liquid jet breakup and droplet formation are suppressed or not observed. Rather, the shear mixing layer density gradient is smeared due to the substantially reduced surface tension and "finger-like" structures are observed, and the injection process is better characterized as gas-gas mixing.

In most previous supercritical injection experiments the fuel was injected at subcritical temperatures but supercritical pressures. For hypersonic flights more-and-more stringent thermal requirements are being placed on aircraft component materials [17]. Specifically, for gas turbine components that are in direct contact with the combustor, use of a regenerative endothermic fuel as coolant for on-board heat sources has been proposed to meet stringent cooling requirements [17]. During the thermal cracking process, the fuel is readily heated up to its supercritical state, so future aircraft are expected to operate with fuels at supercritical pressure and temperature conditions [17]. Similarly, when regenerative fuel is injected beyond its thermodynamic critical point, there is no defined boundary between the injectant and the ambient air. However, undesired condensation could occur under certain injection conditions. Wu et al. [18] experimentally studied injection of pure ethylene near its Body 
thermodynamic critical point into a nitrogen environment. Both shadowgraphs and Schlieren images directly showed that an opaque region existed at the nozzle exit and condensation had occurred. In fact, a liquid core, which generally exists for a liquid jet, was observed even for supercritical gas jet injection. It has also been found that the opaque region can persist from the nozzle exit to a distance as much as 40 nozzle diameters downstream [18], or even 50 nozzle diameters [19]. Effects of back pressure and injection temperature on the occurrence of condensation and the length of the opaque region have also been studied in Ref. [18]. The condensing region was seen to disappear when the injection temperature increased. When this occurred, the supercritical jet was observed to have similar shock structures as those of highly underexpanded ideal-gas jets in terms of barrel shock and Mach disc features. On the other hand, for ambient pressures from atmospheric pressure to 4.1 bar, the length of the opaque region is increased with increasing ambient pressure, while it decreases when the ambient pressure exceeded 4.1 bar. These experimental observations show that even when the fuel is injected at supercritical conditions, a finite length liquid core can exist, which is undesired because condensation could delay gas phase chemical reactions and hence affect the performance of combustor.

Lin et al. [20] also examined the injection of a supercritical binary methane/ethylene fuel, which was thought to be a better representation of jet fuel. An opaque region composed of condensed fuel was observed immediately outside the nozzle from the shadowgraph images, indicating that phase change even had already occurred inside the nozzle before the fuel was issued. This deduction was further verified by their experiments on the injection of supercritical ethylene using a transparent nozzle [20]. Some of the experimental cases were simulated using a homogeneous phase equilibrium model [21, 22] with classical nucleation theory [21]. Doungthip et al. [23] studied supercritical injection behavior of aviation jet fuel under conditions both experimentally (using Schlieren imaging) and numerically. Consistent with the experiments, the penetration length of the high density fluid was found to decrease with increasing fuel temperature. In addition, the spreading angle was found to increase with mass flow rate. Pure n-decane was used as jet fuel physical surrogate in their simulations, as it has critical temperature and pressure similar to that of the JP-8 fuel used in the experiments. The simulations were found to predict the jet spreading angle and penetration length reasonably well compared to the experiments. However, phase change (e.g., fuel condensation) was not considered in their work.

Comprehensive reviews that examine supercritical fuel injection behavior from the perspective of simulations are available. Yang [24] pointed out that flow dynamics and Body 
transport processes under supercritical conditions exhibited rather distinct behaviors compared to those at low pressures due to the introduction of thermodynamic non-ideality and transport anomalies. Bellan [25] presented a critical review of the peculiarities of fluid dynamics, thermodynamics and transport properties at supercritical conditions. Since the isolated droplet evaporation model is widely used in spray simulations, the evaporation process under ambient conditions that are supercritical with respect to fuel critical point is also crucial to accurately simulate the two-phase flows. A related and detailed review was given by Givler and Abraham [26].

Specifically, past research has focused on the following two aspects. The first is transcritical evaporation of single-component and multi-component droplets [27-32]. In all these works, thermodynamic properties were calculated using realistic equation of state (EOS) models to account for high pressure effects and the cubic EOS model had been widely used, but different methods are used to determine the transport properties (e.g., thermal conductivity, diffusion coefficient). It should be noticed that the "critical mixing state" concept has been widely accepted and used in a number of studies [29, 30, 33-35]. The second is to characterize turbulent mixing boundary layers. As mentioned above, the turbulent-injection induced shear forces introduce a mixing layer between the injectant and the ambient gas. There are a number of classical direct numerical simulation (DNS) studies on the development of mixing layers under supercritical injection conditions [36-39]. A recent DNS study [40] further investigated the temporal mixing layer development at supercritical pressure conditions for 5 species using $\mathrm{H}_{2} \mathrm{O}, \mathrm{CO}_{2}, \mathrm{O}_{2}$ and $\mathrm{N}_{2}$ for the upper stream, and $\mathrm{H}_{2} \mathrm{O}, \mathrm{CO}_{2}$ and n-heptane for the lower stream. Inclusion of $\mathrm{H}_{2} \mathrm{O}$ and $\mathrm{CO}_{2}$ was used to simulate the effects of exhaust gas recirculation, which is a common technique used to reduce NOx emissions in automotive engines. It was found that the smallest scales were determined by the variation of the scalars (e.g., thermodynamic and transport properties), and not due to fluid dynamics effects. Another interesting point revealed by the high fidelity simulations was that spinodal decomposition of $\mathrm{H}_{2} \mathrm{O}$ and $\mathrm{CO}_{2}$ was possible, which was supported by the appearance of a negative effective diffusivity, meaning that these two species tend to separate from the other species. In addition, it was shown that the necessary and sufficient condition for this decomposition was the presence of the two species in both streams and they did not need to be uniformly distributed initially.

\section{Objective}

Roy et al. [41] performed experiments on injecting a supercritical fuel into a nitrogen 
environment using planar laser induced fluorescence (PLIF). The experiments show that condensation occurred and was evidenced via observable small droplet-like structures. This was not expected because both the injectant and ambient were at supercritical states and phase change "should not" occur under such conditions. At the same time, the experiments showed that condensation occurred only when the temperature difference between the injectant and ambient was large. The current work aims to provide some insights into the underlying physics associated with the observed phase transition process using computational fluid dynamics (CFD). To our knowledge, this is the first time that these experiments have been simulated. The paper is organized as follows. The fluid and thermodynamic solvers are first introduced to formulate the general simulation framework. Phase behavior is then analyzed from thermodynamic calculations. CFD simulations are then used to investigate the supercritical injection and mixing processes, and the results are compared with the available experimental data. The paper ends with a summary and conclusions.

\section{Model formulation}

\subsection{The fluid solver}

The open-source multi-dimensional CFD code KIVA-3V Release 2 [42] was used. This code has been widely used in diesel spray combustion simulations (see for example Refs. [43, 44]). The compressible flow solver in the KIVA code solves the following Reynolds-average Navier-Stokes (RANS) equations [45] while neglecting gravity, spray and chemistry effects:

$$
\left\{\begin{array}{l}
\frac{\partial \rho_{i}}{\partial t}+\nabla\left(\rho_{i} \vec{V}\right)=\nabla\left(\rho \mathcal{D} \nabla\left(\frac{\rho_{i}}{\rho}\right)\right) \\
\frac{\partial \rho \vec{V}}{\partial t}+\nabla(\rho \vec{V} \vec{V})=-\nabla P-\nabla\left(\frac{2}{3} \rho k\right)+\nabla J \\
\frac{\partial(\rho u)}{\partial t}+\nabla(\rho u \vec{V})=-P \nabla \vec{V}-\nabla F+\rho \varepsilon
\end{array}\right.
$$

$\rho$ is the mixture density, $\mathcal{D}$ is the diffusion coefficient, $\vec{V}$ is the velocity vector, $u$ is the specific internal energy and $P$ is the pressure. $k$ and $\varepsilon$ are the turbulent kinetic energy (TKE) and its dissipation rate, respectively. $J$ is the viscous stress tensor calculated using

$$
J=\mu\left[\nabla \vec{V}+(\nabla \vec{V})^{T}\right]-\lambda \mathbf{I} \nabla \vec{V}
$$

$\mu$ is the dynamic viscosity coefficient. $F$ is the heat flux due to heat conduction and species diffusion:

$$
F=-K \boldsymbol{\nabla} T-\rho \mathcal{D} \sum_{i} h_{i} \boldsymbol{\nabla}\left(\frac{\rho_{i}}{\rho}\right)
$$

where $h_{i}$ is the specific enthalpy of species $i$ and $K$ is the heat conduction coefficient . 
The standard KIVA code uses the ideal gas relation. To account for high pressure thermodynamic non-idealities, the code was modified to account for real gas effects. More details can be found in Refs. [46, 47]. The Peng-Robinson (PR) EOS [48] was adopted due to its good balance between accuracy and efficiency for practical CFD simulations, though in principle any other complex EOS model could be used. Zhu and Aggarwal [31] found that PR EOS gave better results than other cubic EOS models in studying the high pressure droplet evaporation. Other research applying the PR EOS to study multiphase flows can be found in Refs. [21, 32, 36, 40, 49], and details about the PR EOS can be found in Appendix A. It should be noted here that the binary interaction parameter, which is the only parameter for the EOS model, needs to be tuned with available vapor-liquid equilibrium (VLE) data for the mixtures of interest.

\subsection{The thermodynamic solver}

Thermodynamic equilibrium is assumed at the end of the hydrodynamics simulation steps to determine the fluid state of each computational cell. For standard ideal gas simulations, the cell temperature is first calculated from the internal energy, which is assumed to be a single function of temperature. The pressure is then determined from the ideal gas law. When real gas effects are considered like in the current work, there are two extra considerations: (1) thermodynamic calculations must be modified since the internal energy depends on both temperature and pressure; (2) phase stability must be further considered. In particular, after the fluid dynamics simulation advances the internal energy and species masses $\left(u\right.$ and $\left.\rho_{i}\right)$ in each computational cell in time, the thermodynamic solver must be applied to determine the state with potential phase separation. From classical thermodynamics, for a system with specified internal energy, volume and mole number of each component, the thermodynamic equilibrium state has the maximum entropy [50]. This equilibrium calculation is named isoenergetic-isochoric flash (UVn flash). Therefore, the goal of the UVn flash computation is to determine the maximum entropy $(S)$ state of a system (i.e., each numerical cell) with a given set of specified inputs: total mixture internal energy $\left(U^{s p e c}\right)$, total volume $\left(V^{s p e c}\right)$, and

mole numbers of each component $\left(n_{i}^{\text {spec }}\right)$. Following this principle, a direct entropy maximization method was formulated and developed following the method of [51]. In this method, the objective function

$$
f_{o b j}=-S(U, V, \vec{n}) / R_{u}
$$

is to be minimized with the following constraints: 


$$
\left\{\begin{array}{l}
\sum_{j} U_{j}=U^{\text {spec }} \\
\sum_{j} V_{j}=V^{\text {spec }} \\
\sum_{j} n_{i j}=n_{i}^{\text {spec }}, i=1, \ldots, N_{c}, j=1, \ldots, N_{P}
\end{array}\right.
$$

$\vec{n}$ is the mole number array for a multicomponent mixture. $N_{c}$ and $N_{P}$ are the numbers of components and phases, respectively.

The phase equilibrium solver solves the set of nonlinear equations as described by Qiu et al. [52]. A Newton-type second-order method [53] was applied to find the maximum entropy state. Changes to the Hessian matrix were made when necessary to ensure positive-definiteness and hence the convexity, and a line search algorithm was applied to determine the maximum step size along the descending direction. More details can be found in Refs. [54-57] and Refs. [51, 58].

There are several points worth noticing in the above treatment. First, by incorporating a realistic EOS model (i.e., PR EOS), real gas effects are considered in a consistent way with the fluid solver, realizing a unified treatment throughout the simulations. Second, with the application of the phase stability test based on Gibbs free energy minimization [59], potential phase separation (Consideration (2) above) is rigorously considered. Third, while the temperature and pressure of each phase can be different during the iterations, they will be the same when convergence is obtained, indicating an equilibrium state has been found (Consideration (1) above). Finally, because there is no time scale associated with phase change from the thermodynamic calculations, phase transition is achieved instantaneously. Verification of this assumption is presented in Appendix B.

A sizable number of thermodynamic properties need to be calculated during the numerical calculations. Since the PR EOS is explicit in pressure, derivatives with temperature, volume and mole numbers as independent variables are preferred. In contrast, the direct entropy maximization method above needs derivatives with internal energy, volume and mole numbers as independent variables. Such transformation for different partial derivatives needed in the Hessian matrix can be preceded using the Jacobian transformation detailed in the Appendices A and B in Ref. [51].

In the case of a pure species the entropy maximization requires solving the following set of non-linear equations $[21,52,60]$

$$
\left\{\begin{array}{l}
\lambda \rho_{V} u_{V}+(1-\lambda) \rho_{V} u_{L}=\rho^{s p e c} u^{\text {spec }} \\
\lambda=\left(\rho^{\text {spec }}-\rho_{L}\right) /\left(\rho_{V}-\rho_{L}\right) \\
P=P^{s}(T) \\
f\left(T, P, Z_{V}\right)=f\left(T, P, Z_{L}\right)
\end{array}\right.
$$


When an EOS model is used, it is convenient to calculate the thermodynamic properties, such as the specific internal energy, enthalpy and entropy, using the departure functions:

$$
\left\{\begin{array}{l}
u=u^{0}+u^{d} \\
h=h^{0}+h^{d} \\
S=S^{0}+S^{d}
\end{array}\right.
$$

The superscript "o" and "d" stand for the ideal gas and departure properties, respectively. In this work, the reference state was taken at $298.15 \mathrm{~K}$ and 1 bar. The departure functions are determined using relationships in Refs. [61, 62]

$$
\left\{\begin{array}{l}
u^{d}=\int_{\infty}^{v}\left[T\left(\frac{\partial P}{\partial T}\right)_{v}-P\right] d v \\
h^{d}=u^{d}+R_{u} T(Z-1) \\
S^{d}=R_{u} \ln Z+\int_{\infty}^{v}\left[\left(\frac{\partial P}{\partial T}\right)_{v}-\frac{R_{u}}{v}\right] d v
\end{array}\right.
$$

The compressibility factor $(Z)$, an indication of departure from ideal gas behavior, is calculated using

$$
Z=P /\left(\rho R_{u} T\right)
$$

For the ideal gas, $Z=1$. For multicomponent mixtures at high-pressure conditions, the partial molar enthalpy $\left(h_{i}\right)$ is calculated according to

$$
\frac{h_{i}-h_{i}^{o}}{R_{u} T}=-T \frac{\partial \ln \left(\varphi_{i}\right)}{\partial T}
$$

The specific heat ratio $(\gamma)$ is defined as:

$$
\gamma=\frac{C_{P}}{C_{V}}
$$

The isobaric and isochoric specific heat ratios, $C_{P}$ and $C_{V}$, are related by

$$
C_{P}=C_{V}+T\left(\frac{\partial P}{\partial T}\right)_{v}\left(\frac{\partial v}{\partial T}\right)_{P}
$$

In addition, the isentropic sound speed $\left(C_{s}\right)$ is calculated using the relation:

$$
C_{S}^{2}=\left(\frac{\partial P}{\partial \rho}\right)_{S}=\left(\frac{\partial P}{\partial \rho}\right)_{T}+\frac{T}{C_{v} \rho^{2}}\left[\left(\frac{\partial P}{\partial T}\right)_{\rho}\right]^{2}
$$

\section{Thermodynamic analysis and phase behavior}

In the experiments of Roy et al. [41], fluoroketone $\left(\mathrm{C}_{6} \mathrm{~F}_{12} \mathrm{O}\right)$ was used as the injectant. It was selected due to its favorable lower critical point, so it is easier to attain the desired supercritical conditions. At the same time, it is safe to use as it is not flammable. It has also been used as the injectant to study supercritical elliptical jet disintegration characteristics [63]. However, the availability of the thermodynamic properties of this species is rather limited. Tuma [64] noticed that the perfluorohexane $\left(\mathrm{C}_{6} \mathrm{~F}_{14}\right)$ has similar properties to those of 
fluoroketone, such as the critical point, latent heat and surface tension at normal conditions. Indeed, a comparison of the density contours of pure $\mathrm{C}_{6} \mathrm{~F}_{12} \mathrm{O}$ and $\mathrm{C}_{6} \mathrm{~F}_{14}$ is shown in Fig. 1 using the PR EOS with increments of $5 \mathrm{~K}$ and 1 bar for temperature and pressure, respectively. For a wide range of conditions, it is seen that the two species show similar P-T- $\rho$ relationships. The critical properties are summarized in Table 1 , where those of pure $\mathrm{C}_{6} \mathrm{~F}_{14}$ were obtained from the DIPPR database [65]. Notice that the acentric factor for $\mathrm{C}_{6} \mathrm{~F}_{12} \mathrm{O}$ is not found in the literature and hence it is estimated using the saturation pressure at normal conditions [66]. Realizing that ideal gas properties of $\mathrm{C}_{6} \mathrm{~F}_{14}$ can be calculated using the NASA 7-coefficient polynomials [67], perfluorohexane was used as the injectant in the present study.

Since the phase transition is mainly determined from the thermodynamics calculations, capturing the phase behavior of the two pure species and their mixture is necessary for the CFD simulations. VLE calculations of pure $\mathrm{C}_{6} \mathrm{~F}_{14}$ were first performed, and the predicted saturation pressure line is shown in Fig. 2(a). The overall match is very good for the entire range of conditions (i.e., from the triple to the critical points) of two-phase mixture. Also, the predicted linear relationship between $\log (\mathrm{P})$ and 1000/T agrees well with the classical Clausius-Clapeyron relation.

The VLE of the binary mixture of $\mathrm{C}_{6} \mathrm{~F}_{14}$ and $\mathrm{N}_{2}$ was also considered. As experimental VLE data for such mixtures was not found in the literature, and the interaction parameter is not expected to be zero for such a pair of a large and a small species, the interaction parameter for $\mathrm{C}_{6} \mathrm{~F}_{14}$ and $\mathrm{CO}_{2}$ mixtures was used, and use of 0.064 as the interaction parameter yields good agreement with available experimental data found in Ref. [68], as discussed in Appendix C.

Due to the non-ideal behavior at high pressure conditions, the latent heat of vaporization also departs from the ideal value [29, 31, 54], as quantified by Eq. (10) and illustrated in Fig. 2(b). It is noticed that the latent heat is decreased at higher pressures for a given temperature. Therefore, the progressively reduced heat of vaporization means that the energy barrier to cross for a liquid-vapor transition process is decreased at higher pressures and it becomes null above the mixture critical point. Behavior near the critical point is also shown in Fig. 3, which shows comparisons of density and isobaric specific heat capacity $\left(C_{p}\right)$ with NIST data [69]. Non-monotonic behavior of $C_{p}$ is observed near the critical temperature in both real gas calculations and referenced experimental data, which is not captured by the linear relationship in ideal gas calculations. This means that the properties near the critical point are very sensitive to a small change of temperature. Though the difference between ideal and real 
gas calculations diminishes at higher temperatures, their discrepancy is still large for $\mathrm{C}_{6} \mathrm{~F}_{14}$ considered here.

Figure 4(a) shows the pressure-mole fraction phase diagram of the binary mixture at three temperatures at pressures from 10 to 100 bar. It is seen that both pressure and temperature exhibit non-linear effects on phase behavior. Particularly, while $\mathrm{C}_{6} \mathrm{~F}_{14}$ in the gas phase shows a systematic change with temperature, the mole fraction of $\mathrm{C}_{6} \mathrm{~F}_{14}$ in the liquid phase does not. More specifically, for pressures below about 22 bar, liquid $\mathrm{C}_{6} \mathrm{~F}_{14}$ mole fraction increases with temperature, but the trend is opposite when the pressure is higher. It is also noticed that even though the critical pressure of $\mathrm{C}_{6} \mathrm{~F}_{14}$ is less than 20 bar, a two-phase region still exists at higher pressures (between 20 and 100 bar). Evidently the mixture phase behavior is quite different from that of the two pure species (for which the two-phase region is on the saturation pressure line), indicating that mixing plays an important role, and it is also quite non-linear. This has also been seen for alkane and nitrogen mixtures with very different molecule structures, such as n-heptane/ $\mathrm{N}_{2}[70]$ and n-octane/ $\mathrm{N}_{2}$ [71]. Furthermore, retrograde condensation behavior is seen for the mixture. For example, Fig. 4(b) shows the phase transition during the heating process for the 70:30 mixture at 60 bar. It is seen that initially the heat addition increases the vapor phase due to evaporation, which is expected. Nonetheless, after the minimum liquid phase point at around $85 \%$ at $295 \mathrm{~K}$, further heating does not enhance the evaporation process - as would be expected-but counter-intuitively condensation occurs.

Two important thermodynamic properties, the isentropic sound speed and the specific heat ratio, are shown in Fig. 5 at three mixture compositions and pressures (at the relevant experimental conditions below). The sound speed of each phase is calculated after the equilibrium composition of each phase is determined using the phase equilibrium solver, as detailed in Ref. [54]. (Determination of the sound speed of a two-phase mixture, rather than that of each phase, can be performed using the methodologies detailed in Refs. [72-74].) First, it is noted that both $C_{s}$ and $\gamma$ of the liquid phase are higher than that of the vapor phase, which is also known behavior for pure species. However, since the two phases can have very different compositions (e.g., higher $\mathrm{C}_{6} \mathrm{~F}_{14}$ in the liquid phase but higher $\mathrm{N}_{2}$ composition in the vapor phase) and compressibility factors, differences in sound speed of the two phases are not as large as those for pure species [57]. Second, Figures 5(b) and (d) show non-monotonic behavior near the critical point of pure $\mathrm{C}_{6} \mathrm{~F}_{14}$. For mixtures with high $\mathrm{C}_{6} \mathrm{~F}_{14}$ ratio, $\gamma$ is found to be bifurcated at around $450 \mathrm{~K}$, as also observed for $C_{P}$ in Fig. 3. Third, for the two-phase 
mixture, both $C_{S}$ and $\gamma$ of the two phases increase with pressure, but decrease with temperature. In the single phase state, pressure seems to play a negligible role on $C_{s}$ for the three mixtures considered, but it increases with temperature. Finally, the pressure effect on $\gamma$ decays as the $\mathrm{C}_{6} \mathrm{~F}_{14}$ composition increases.

\section{Results and discussions}

A 0.5 degree 2-D axisymmetric mesh was used in the current study, as shown in Fig. 6. The experimental injector diameter is $2 \mathrm{~mm}$ and 10 grid points were evenly distributed across the nozzle radius. However, outside the injector, there were 32 and 50 gradually increasing grid points in the radial and axial directions, respectively. The mesh was found to yield adequately grid-independent results. The computational domain had a radius and height of 4 and $12 \mathrm{~cm}$, respectively. Velocity inflow and continuative outflow boundary conditions were imposed at the nozzle exit and the bottom of the chamber, respectively. Injection starts at the beginning of the simulations. Adiabatic and no-slip wall boundary condition were applied at all the walls. Initially the chamber is filled with nitrogen and the flow there was quiescent. The standard $k-\varepsilon$ turbulence model was used.

\subsection{Supercritical-to-supercritical injection}

All eight experimental conditions in Ref. [41] are summarized in Table 2 and shown schematically in Fig. 7. The case indices are the same as in the experiments. In the following, unless otherwise specifically noted, "supercritical" is used with respect to the critical properties of the injectant (i.e., $\mathrm{C}_{6} \mathrm{~F}_{14}$ ), but not the thermodynamic critical point of a mixture (e.g., $\mathrm{C}_{6} \mathrm{~F}_{14}$ and $\mathrm{N}_{2}$, as calculated above). It is seen from Table 2 that for Cases 1-8, (1) both injection and chamber pressures are supercritical; (2) injection temperature is supercritical but the chamber temperature is subcritical for some cases. Therefore, in the following discussions, "supercritical" and "subcritical" are further used to represent the state difference in temperature. In addition, the calculations show that the injection Mach number is less than unity for all cases and the injection Reynolds number is of the order of $1.0 \mathrm{e}+05$. In addition, 2 other cases (i.e., Cases 9 and 10) were added to aid the analysis of phase changes to be discussed below.

Supercritical-to-supercritical injection (i.e., Cases 5-8) is first considered. Simulation results are shown along with the experimental data of the injectant density in Figs. 8-11. It should be mentioned that only the part of the domain that had optical access is shown. Also notice that for Case 5, because the injectant is just at a liquid-like state close to the critical 
point, the density is quite large compared to the other cases. To show the importance of real gas effects at supercritical conditions, ideal gas simulations are also presented. For all the comparisons, unless otherwise specifically noted, physical dimensions and magnitudes of the colorbars are the same.

The overall comparison shows that the ideal gas simulations generally underestimate the peak values of the injectant density for all the cases. In contrast, significant improvements are observed when real gas effects are considered. The fluid thermodynamic state is better represented, so the predicted density field is better predicted and the jet core length agrees better with the experiments from direct visualization. For instance, the experimental data for Case 6 in Fig. 9(a) indicates that the last pocket of high density region (about $180 \mathrm{~kg} / \mathrm{m}^{3}$ ) is located about $35 \mathrm{~mm}$ downstream of the nozzle exit. The ideal gas simulation underestimates the distance to at around $23 \mathrm{~mm}$. In contrast, the real gas simulation in Fig. 9(c) predicted the core length of about $33 \mathrm{~mm}$. For a better comparison density profiles at two distances downstream of the nozzle exit, namely 15 and $35 \mathrm{~mm}$, are shown in Fig. 12. Consistent with the observations, the real gas prediction is generally closer to the experiments, especially in the far field.

Discrepancy of the jet spreading angle is also noticed. In comparison, the real gas simulation generally shows a smaller jet spreading angle than the ideal gas. Classical gas jet mixing theories generally show that the spreading angle depends on the density ratio between the ambient and the injectant, $\rho_{a} / \rho_{f}$. This is consistent with the predictions from the present ideal and real gas simulations. Due to the underestimated $\rho_{f}$ from the ideal gas relation, the spreading angle becomes larger compared to that of real gas simulation. Some more analysis on the global parameters, including the spreading angle and core length for supercritical jets, can be found in Refs. $[75,76]$ and the references therein. It is also noticed that the current RANS simulations are not able to capture wavy structures in the mixing layer, which would require more complicated turbulence modeling. In addition, when compared to the experimental images, both ideal and real gas simulations show smaller spreading angle. Several factors can be accounted for this inadequacy. First, to a large extent it is believed to be related to the uncertainty in the inlet boundary conditions (e.g., fluctuations of inlet velocity and pressure) as nozzle outlet flow is turbulent. Second, initial chamber turbulence level could affect the jet evolution as it is hard to determine in the experiments. Third, modeling assumptions and parameters in the RANS simulation could also play an important role so more detailed simulations are needed. 


\subsection{Supercritical-to-subcritical injection}

Real gas simulation results of Cases 1-4 for supercritical-to-subcritical injection are shown along with the experimental data in Figs. 13-16, respectively. A direct comparison of the injectant density at two axial locations (i.e., 10 and $20 \mathrm{~mm}$ ) is shown in Fig. 17. The predictions are found to be similar to those for the supercritical-to-supercritical injection.

During the real gas simulations the stability of the one-phase state solution in each computational cell is tested with the developed phase stability solver [54]. If instability of a single phase is detected, phase change (from gas to liquid) occurs instantaneously and the phase transition event is captured by the thermodynamic solver discussed in Section 3.2. The simulations showed that phase change was not possible for the supercritical-to-supercritical injections. Furthermore, condensation was predicted only for Case 1 for a supercritical-to-subcritical injection, as also observed in the experiments [41].

Figure 18 shows jet evolution at 3,5 and 9ms after the start of injection (ASI) for Case 1. Injectant mass fraction contour (Figs. 18 (a)-(c)) is used to better illustrate the jet mixing layer, and they show that a quasi-steady state has been achieved around 5ms. The contour plot of the liquid volume fraction is shown in Figs. 18 (d)-(f). It is seen that condensation occurs only in the jet periphery where there is a small portion of injectant but with abundant entrained nitrogen. However, the maximum liquid volume fraction is about $0.022 \%$, meaning that the accompanying condensation event is feeble and the two-phase mixture is close to the dew point line. As time evolves, the condensation region is found to penetrate further downstream of the jet and it spreads outward.

To better quantify the effects of chamber temperature alone, additional 2 cases, Cases 9 and 10, were considered. The only difference is that the chamber temperature was reduced from Case $9\left(\operatorname{Tr}_{\text {chamber }}=0.8\right)$ to Case $10\left(\operatorname{Tr}_{\text {chamber }}=0.6\right)$. Direct comparison with Cases 1 $\left(\operatorname{Tr}_{\text {chamber }}=0.69\right)$ is shown in Fig. 19 at $7 \mathrm{~ms}$ ASI. The turbulent kinetic energy (TKE) in Fig. 19 (a)-(c) shows that in all three cases, the turbulence is similar, which is expected as the injection conditions are the same. The temperature contours in Fig. 19 (d)-(f) show that the decrease in chamber temperature increases the temperature gradient, and hence heat transfer between the "hot" injectant and "cold" nitrogen is intensified. The direct consequence is enhanced condensation, as observed from the increased liquid volume fraction shown separately in Fig. 20. Notice that the scales of the colorbars are different from Case 1 to Case 10. The maximum liquid volume fraction is $0.18 \%$ for Case 10 , which is about a five-fold increase from Case 1. 
It is helpful to monitor the state evolution in a numerical cell. For better illustration, Points A and B with coordinates $(1.9 \mathrm{~mm}, 5.06 \mathrm{~mm})$ and $(3.2 \mathrm{~mm}, 15.03 \mathrm{~mm})$ in the $r-z$ plane were selected (as also shown in Fig. 20 (a)), corresponding to 5 and $15 \mathrm{~mm}$ downstream of the nozzle, respectively. Temperature and liquid phase mole fraction are shown in Fig. 21 for the three cases. Compared to Case 9 without condensation, due to the decrease of temperature, condensation is seen in Case 1 and it is actually boosted for Case 10, which is shown by the elevated liquid mole fraction and is consistent with Fig. 20. For the temperature plot, the first increase is due to the mixing of high temperature injectant. At both locations, temperature tends to decrease as heat is transferred to the surrounding low temperature ambient, until a nearly steady state is achieved. This is also true for the liquid mole fraction, for which the quasi-steady value is about $0.2 \%$ and $0.5 \%$ for Points $\mathrm{A}$ and $\mathrm{B}$, respectively. It is found that condensation is advanced from $1.2 \mathrm{~ms}$ ASI for Case 1 to $0.3 \mathrm{~ms}$ ASI for Case 10 at Point A. In comparison, a condensation event takes place at Point B at 3.0 and $1.2 \mathrm{~ms}$ ASI for Case 10 and Case 1, respectively. It is also noticed there are two condensation events at Point B. The first one does not last long, as the jet is still developing. In contrast, the second condensed phase is more stable when the thermodynamic conditions there are relatively steady. In addition, the magnitudes of the local property fluctuations are different. Compared to Point A (much closer to the inlet), the perturbations of temperature and liquid mole fraction become less at Point $\mathrm{B}$. The calculations also show that $\mathrm{C}_{6} \mathrm{~F}_{14}$ is the predominant component in the liquid phase (more than 90\%), which is expected and consistent with Fig. 4 (a).

These results show that condensation in the supercritical conditions is only possible when the temperature difference between the injectant and environment is large enough, which is consistent with the conclusions of Ref. [41]. The current simulations do not show condensation in Cases 2 and 3 (while a small number of droplets were observed in the experimental images), which could be due to the use of a different injectant and simplified experimental conditions. Nonetheless, the essential physics is captured by the current modeling approach. Because no condensation was observed in the jet central region where there is only $\mathrm{C}_{6} \mathrm{~F}_{14}$, nor does it occur outside the jet where there is only $\mathrm{N}_{2}$, the phase change is restricted to the turbulent mixing layer. Comparing the contours of liquid volume fraction with the TKE coutours, a correlation between the liquid volume fraction and local turbulence in the condensation region is observed. The higher the local TKE, the higher the liquid volume fraction. Similar results are found in Fig. 21: the lower the temperature, the higher the liquid mole fraction. This discovery is closely related to the phase behavior of a binary mixture. As already detailed in Section 4, mixing plays a determining role in the mixture 
phase behavior, along with temperature and pressure.

In summary, the above observations confirm the important connection between mixing (related to injection parameters) and thermodynamics (related to chamber thermal conditions). Though mixing is important to promote heat, mass and momentum transfers between the "hot" injectant and "cold" ambient, it cannot trigger phase change if mixture is not condensable when the thermodynamic conditions are not suitable. This is supported by the absence of condensation in Case 4 with relatively higher injection velocity and the occurrence of condensation in Case 1. Second, given the similar turbulence levels, only if the chamber temperature is low enough will condensation occur, as evidenced by the comparison in Fig. 20. It is hence concluded that the thermal conditions of the injectant and ambient, and especially the relative difference between them, is another prerequisite for possible phase separation and hence it is as crucial as mixing for possible condensation. Finally, the present phase equilibrium model captures the critical physics associated with phase change occurring under supercritical injection conditions, so it can be used with confidence as a starting point for further analysis.

\section{Conclusions}

Computational fluid dynamic simulations were performed to investigate supercritical injection processes and accompanying potential phase changes. The KIVA $3 \mathrm{v}$ release 2 open source code was modified using the Peng-Robinson equation of state to account for real gas effects in the fluid solver and phase change in the thermodynamic solver. The phase transition model is composed of phase stability and equilibrium calculations based on fundamental thermodynamic principles (i.e., entropy maximization and Gibbs free energy minimization). The simulations were compared with available experimental injectant density data in Ref. [41]. To our knowledge, this is the first simulation of these experiments, and the simulation results are found to agree with some conclusions drawn from the experiments. Specifically, the following observations were found for supercritical-to-supercritical injections (Cases 5-8):

(1) Compared to the experimental data, real gas simulations showed improvements in the prediction of peak density profiles as well as the visible jet core length. However, the spreading angle is somewhat underestimated.

(2) The simulations show that the appearance of a second phase was not possible for all the 4 cases considered, which is consistent with the experiments that showed no droplets. 
Simulations for supercritical-to-subcritical injections (Cases 1-4 and 9-10) showed close connection between mixing and thermodynamics in determining condensation in supercritical injections. The following conclusions were drawn:

(1) The simulations showed that condensation was not observed until the temperature difference between the injectant and the ambient gas was large enough to promote strong enough heat transfer to send the local mixture into the two-phase state. This point is consistent with the discussions and conclusions of the experiments. In the two-phase region, it was shown that the liquid phase fraction was higher if the local temperature was lower and the turbulence intensity was higher.

(2) The simulations predicted that condensation only occurred at the jet periphery, where the local mixing produced a thermodynamically unstable mixture and a second liquid phase appeared to minimize the system Gibbs free energy.

(3) The thermodynamic conditions in the chamber were found to be the other prerequisite to form condensable mixtures to occur and hence it played a critical role for possible phase change along with mixing.

\section{Appendix A. Equation of state model}

The Peng-Robinson EOS has the form

$$
P=\frac{R_{u} T}{v-b}-\frac{a}{v(v+b)+b(v-b)}
$$

$a$ and $b$ are determined using:

$$
\left\{\begin{array}{l}
a=0.457235 \frac{R_{u}^{2} T_{c}^{2}}{P_{c}} \cdot \alpha \\
b=0.077796 \frac{R_{u} T_{c}}{P_{c}} \\
\alpha=\left[1+\kappa\left(1-\sqrt{T / T_{c}}\right)\right]^{2}
\end{array}\right.
$$

with

$$
\kappa=0.37464+1.54226 \omega-0.26992 \omega^{2}
$$

Another formula [77] was used when the acentric factor $\omega$ is greater than 0.5:

$$
\kappa=0.3796+1.485 \omega-0.1644 \omega^{2}+0.01667 \omega^{3}
$$

For a mixture, the one-fluid mixing rule is adopted here:

$$
\left\{\begin{aligned}
a & =\sum_{i} \sum_{j} x_{i} x_{j} a_{i j} \\
b & =\sum_{i} x_{i} b_{i} \\
a_{i j} & =\left(1-\delta_{i j}\right) \sqrt{a_{i}} \sqrt{a_{j}}
\end{aligned}\right.
$$




\section{Appendix B. Thermodynamic limit of phase change}

Thermodynamic equilibrium is usually invoked to determine the state of a single phase mixture. The current phase transition model further considers potential phase separation by minimizing Gibbs free energy. Thus it is of interest to examine if a phase change event could take place during a CFD time step. Well-established theories to determine such time scales do not seem to exist, to our knowledge. However, the characteristic relaxation time required to reach equilibrium can be calculated according to classical homogeneous nucleation theory (see Refs. [78] and [79] and references therein):

$$
\tau^{-1} \approx \frac{2 \xi P x^{l i q} v^{l i q}}{k_{B} T} \sqrt{\frac{\sigma}{2 \pi m}}
$$

where $\tau$ is the characteristic time scale needed to reach steady state; $x^{\text {liq }}$ is the mole fraction of the condensed phase; $\sigma$ is the surface tension of a newly formed nuclei; $m$ is the molecule mass of the condensed species; $v^{\text {liq }}$ is the molecular volume of the condensed phase calculated from saturated liquid density $\rho^{\text {liq,sat }}$. For the maximum time scale, 1 is used for $\xi$ [78]. $\sigma$ is calculated using the formula from DIPPR database [65]:

$$
\sigma=C_{1}\left(1-T / T_{c}\right)^{C_{2}}
$$

where $C_{1}$ and $C_{2}$ are fitting parameters and they are 0.041873 and 1.2059 for pure $\mathrm{C}_{6} \mathrm{~F}_{14}$, respectively.

Calculated time scales at typical conditions (e.g., temperature, pressure and liquid mole fraction) for the current work are tabulated in Table B1, which shows that $\tau$ is typically much less than the hydrodynamic time step (of the order 1.0e-07 s). This validates the current assumption that phase change can be achieved instantaneously. However, it should be noticed that the estimates are based on derivations from self-nucleation theory of a pure species and properties of $\mathrm{C}_{6} \mathrm{~F}_{14}$ are used in the computations. Given the high concentration of $\mathrm{C}_{6} \mathrm{~F}_{14}$ in the condensed phase, these estimates can be used for reference reasonably.

\section{Appendix C. Vapor-liquid equilibria of perfluorohexane and carbon dioxide}

The only parameter for the EOS model is the binary interaction parameter $\left(\delta_{i, j}\right)$. In practice, it is determined with experimental VLE data. Unfortunately, such data for a binary mixture of $\mathrm{C}_{6} \mathrm{~F}_{14}$ and $\mathrm{N}_{2}$ is not found in the literature. As a reasonable substitute for now, the interaction parameter for binary $\mathrm{C}_{6} \mathrm{~F}_{14}-\mathrm{CO}_{2}$ mixtures is used. Thermodynamic properties of 
$\mathrm{CO}_{2}$ are presented in Table 1. Comparison of the predicted VLE with experimental data [68] is presented in Fig. C1 with $\delta_{12}=0.064$. Reasonably good agreement is found for the three temperatures, so this interaction parameter is also used for the mixture of $\mathrm{C}_{6} \mathrm{~F}_{14}$ and $\mathrm{N}_{2}$ in the current work.

\section{Acknowledgements}

The research was conducted at the Engine Research Center (ERC) in the University of Wisconsin-Madison and sponsored by Department of Energy and Sandia National Laboratories through the Advanced Engine Combustion Program (MOU 04-S-383) under the management of Dr. Dennis L. Siebers. Mr. Lu Qiu would like to thank Dr. Yue Wang and Mr. Jae Lim at ERC, for helpful discussions during the initial stage of this work. The authors are also grateful to Dr. Anarb Roy (now at Intel Inc.) for his kind sharing of the experimental data used in this work.

\section{Notation}

$a=$ energy parameter in Eq. (A1)

$a_{i}=$ energy parameter for component $i$ in Eq. (A4)

$a_{i j}=$ energy parameter between component $i$ and $j$ in Eq. (A4)

$b_{i}=$ volume parameter for component $i$ in Eq. (A4)

$b=$ volume parameter in Eq. (A1)

$C_{1}=$ constant used in Eq. (B2)

$C_{2}=$ constant used in Eq. (B2)

$C_{P}=$ isobaric heat capacity

$C_{S}=$ isentropic sound speed

$C_{V}=$ isochoric heat capacity

$f=$ fugacity

$f_{o b j}=$ the objective function in Eq. (4)

$F=$ heat flux term

$h=$ specific enthalpy

$J=$ viscous stress tensor

$k=$ turbulent kinetic energy

$k_{B}=$ Boltzman constant used in Eq. (B1)

$K=$ heat conduction coefficient

$m=$ molecular mass used in Eq. (B1)

$M a=$ the Mach number

$n_{i}=$ mole number of component $i$

$n_{i j}=$ mole number of component $i$ in phase $j$

$n=$ mole number

$N_{c}=$ number of components

$N_{P}=$ number of phases

$P=$ pressure

$R e=$ the Reynolds number

$R_{u}=$ universal gas constant, $8.314 \mathrm{~J} / \mathrm{mol}-\mathrm{K}$

$S=$ entropy

$T=$ temperature

$u=\quad$ specific internal energy 
$U=$ internal energy

$v=$ specific molar volume

$v^{l i q}=$ molecular volume of the liquid phase

$\vec{V}=$ velocity vector

$x^{l i q}=$ mole fraction of the liquid phase

$x_{i}=$ mole fraction of component $i$

$Z=$ compressibility factor

\section{Greek letters}

$\alpha=$ parameter in Eq. (A2)

$\mathcal{D}=$ mass diffusion coefficient

$\varepsilon=$ turbulent dissipation rate

$\gamma=$ specific heat ratio

$\delta_{i, j}=$ binary interaction parameter between component $i$ and $j$

$\kappa=$ parameter in Eq. (A3)

$\lambda=$ vapor phase volume fraction

$\tau=$ characteristic time scale to reach equilibrium

$\mu=$ dynamic viscosity coefficient

$\xi=$ constant used in Eq. (B1)

$\rho=$ density

$\sigma=$ surface tension

$\varphi=$ fugacity coefficient

$\omega=$ acentric factor

\section{Subscripts}

$a=$ ambient species

$c=$ critical property

$f=$ injectant species

$L=$ liquid phase

$V=$ vapor phase

\section{Superscripts}

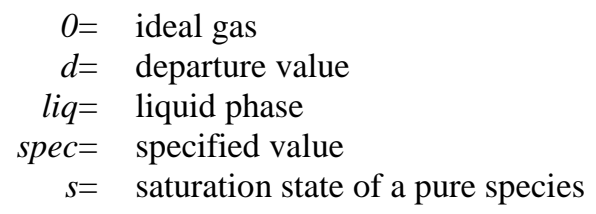

\section{Abbreviations}

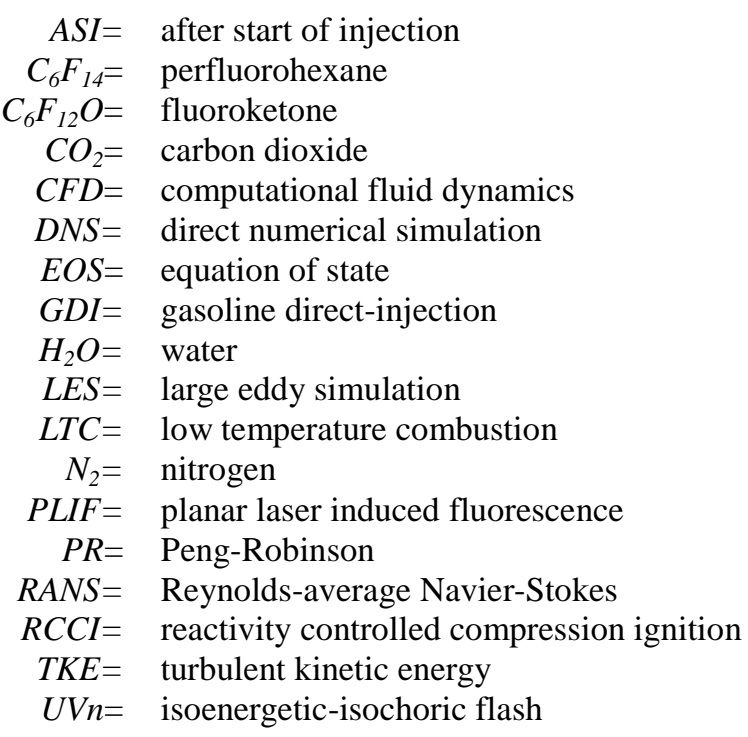


$V L E=$ vapor-liquid equilibrium

\section{References}

[1] R.D. Reitz, Atomization and other breakup regimes of a liquid jet, $\mathrm{PhD}$ Thesis, Princeton University, 1978.

[2] R.D. Reitz, Modeling atomization processes in high-pressure vaporizing sprays, Atomization and Spray Technology, 3 (1987) 309-337.

[3] J.C. Beale, R.D. Reitz, Modeling spray atomization with the Kelvin-Helmholtz/Rayleigh-Taylor hybrid model, Atomization Spray, 9(6) (1999) 623-650.

[4] V. Vuorinen, M. Larmi, E. Antila, O. Kaario, E. El-Hannouny, S. Gupta, Near Nozzle Diesel Spray Modeling and X-Ray Measurements, SAE 2006-01-1390.

[5] F. Tao, P. Bergstrand, Effect of Ultra-High Injection Pressure on Diesel Ignition and Flame under High-Boost Conditions, SAE 2008-01-1603.

[6] M. Jia, Z. Peng, M. Xie, Evaluation of Breakup Models and Application to the Mixture Preparation Process for Diesel HCCI Engines, SAE 2008-01-0023.

[7] S. Som, S.K. Aggarwal, Effects of primary breakup modeling on spray and combustion characteristics of compression ignition engines, Combust Flame, 157(6) (2010) 1179-1193.

[8] S. Srivastava, H. Schock, F. Jaberi, Numerical Simulations of Turbulent Sprays with a Multicomponent Evaporation Model, SAE 2013-01-1603.

[9] H. Wang, R.D. Reitz, M. Yao, B. Yang, Q. Jiao, L. Qiu, Development of an n-heptane-n-butanol-PAH mechanism and its application for combustion and soot prediction, Combust Flame, 160(3) (2013) 504-519.

[10] H. Wang, Q. Jiao, M. Yao, B. Yang, L. Qiu, R.D. Reitz, Development of an n-heptane/toluene/polyaromatic hydrocarbon mechanism and its application for combustion and soot prediction, International Journal of Engine Research, 14(5) (2013) 434-451.

[11] J. Oefelein, R. Dahms, G. Lacaze, Detailed Modeling and Simulation of High-Pressure Fuel Injection Processes in Diesel Engines, SAE Int. J. Engines, 5(3) (2012) 1410-1419.

[12] R.N. Dahms, J.C. Oefelein, On the transition between two-phase and single-phase interface dynamics in multicomponent fluids at supercritical pressures, Physics of Fluids, 25(9) (2013) 092103.

[13] G. Anitescu, T.J. Bruno, L.L. Tavlarides, Dieseline for Supercritical Injection and Combustion in Compression-Ignition Engines: Volatility, Phase Transitions, Spray/Jet Structure, and Thermal Stability, Energy \& Fuels, 26(10) (2012) 6247-6258.

[14] S.L. Kokjohn, R.M. Hanson, D.A. Splitter, R.D. Reitz, Fuel reactivity controlled compression ignition (RCCI): a pathway to controlled high-efficiency clean combustion, International Journal of Engine Research, 12(3) (2011) 209-226.

[15] J.A. Newman, T.A. Brzustowski, Behavior of a Liquid Jet near the Thermodynamic Critical Region, AIAA J, 9(8) (1971) 1595-1602.

[16] B. Chehroudi, Recent Experimental Efforts on High-Pressure Supercritical Injection for Liquid Rockets and Their Implications, International Journal of Aerospace Engineering, 2012 (2012) 31.

[17] E. Tim, USAF supercritical hydrocarbon fuels interests, in: 31st Aerospace Sciences Meeting, American Institute of Aeronautics and Astronautics, 1993.

[18] P.-K. Wu, T.H. Chen, A.S. Nejad, C.D. Carter, Injection of supercritical ethylene in nitrogen, J Propul Power, 12(4) (1996) 770-777.

[19] P.K. Wu, M. Shahnam, K.A. Kirkendall, C.D. Carter, A.S. Nejad, Expansion and mixing processes of 
underexpanded supercritical fuel jets injected into superheated conditions, J Propul Power, 15(5) (1999) 642-649.

[20] K.C. Lin, S.K. Cox-Stouffer, T.A. Jackson, Structures and Phase Transition Processes of Supercritical Methane/Ethylene Mixtures Injected Into A Subcritical Environment, Combustion Science and Technology, 178(1-3) (2006) 129-160.

[21] A.M. Star, J.R. Edwards, K.C. Lin, S. Cox-Stouffer, T.A. Jackson, Numerical simulation of injection of supercritical ethylene into nitrogen, J Propul Power, 22(4) (2006) 809-819.

[22] L. Qiu, Y. Wang, R.D. Reitz, On regular and retrograde condensation in multiphase compressible flows, International Journal of Multiphase Flow, 64(0) (2014) 85-96.

[23] T. Doungthip, J.S. Ervin, T.F. Williams, J. Bento, Studies of injection of jet fuel at supercritical conditions, Ind Eng Chem Res, 41(23) (2002) 5856-5866.

[24] V. Yang, Modeling of supercritical vaporization, mixing, and combustion processes in liquid-fueled propulsion systems, Proceedings of the Combustion Institute, 28(1) (2000) 925-942.

[25] J. Bellan, Supercritical (and subcritical) fluid behavior and modeling: drops, streams, shear and mixing layers, jets and sprays, Prog Energ Combust, 26(4-6) (2000) 329-366.

[26] S.D. Givler, J. Abraham, Supercritical droplet vaporization and combustion studies, Prog Energ Combust, 22(1) (1996) 1-28.

[27] J.A. Manrique, G.L. Borman, Calculations of steady state droplet vaporization at high ambient pressures, Int J Heat Mass Tran, 12(9) (1969) 1081-1095.

[28] J.D. Jin, G.L. Borman, A Model for Multicomponent Droplet Vaporization at High Ambient Pressures, SAE Technical Paper 850264, (1985).

[29] K.C. Hsieh, J.S. Shuen, V. Yang, Droplet Vaporization in High-Pressure Environments .1. Near Critical Conditions, Combustion Science and Technology, 76(1-3) (1991) 111-132.

[30] E.W. Curtis, P.V. Farrell, A Numerical Study of High-Pressure Droplet Vaporization, Combust Flame, 90(2) (1992) 85-102.

[31] G.S. Zhu, S.K. Aggarwal, Transient supercritical droplet evaporation with emphasis on the effects of equation of state, Int J Heat Mass Tran, 43(7) (2000) 1157-1171.

[32] G.S. Zhu, R.D. Reitz, Engine Fuel Droplet High-Pressure Vaporization Modeling, Journal of Engineering for Gas Turbines and Power, 123(2) (2000) 412-418.

[33] H. Jia, G. Gogos, Investigation of liquid droplet evaporation in subcritical and supercritical gaseous environments, Journal of Thermophysics and Heat Transfer, 6(4) (1992) 738-745.

[34] L. Zhang, S.C. Kong, High-pressure vaporization modeling of multi-component petroleum-biofuel mixtures under engine conditions, Combust Flame, 158(9) (2011) 1705-1717.

[35] H. Meng, V. Yang, Vaporization of two liquid oxygen (LOX) droplets in tandem in convective hydrogen streams at supercritical pressures, Int J Heat Mass Tran, 68(0) (2014) 500-508.

[36] N.A. Okong'o, J. Bellan, Direct numerical simulation of a transitional supercritical binary mixing layer: heptane and nitrogen, Journal of Fluid Mechanics, 464 (2002) 1-34.

[37] N. Okong'o, J. Bellan, Real-gas effects on mean flow and temporal stability of binary-species mixing layers, AIAA J, 41(12) (2003) 2429-2443.

[38] J. Bellan, Theory, modeling and analysis of turbulent supercritical mixing, Combustion Science and Technology, 178(1-3) (2006) 253-281.

[39] L.C. Selle, N.A. Okong'o, J. Bellan, K.G. Harstad, Modelling of subgrid-scale phenomena in supercritical transitional mixing layers: an a priori study, Journal of Fluid Mechanics, 593 (2007) 57-91.

[40] E. Masi, J. Bellan, K.G. Harstad, N.A. Okong'o, Multi-species turbulent mixing under 
supercritical-pressure conditions: modelling, direct numerical simulation and analysis revealing species spinodal decomposition, Journal of Fluid Mechanics, 721 (2013) 578-626.

[41] A. Roy, C. Joly, C. Segal, Disintegrating supercritical jets in a subcritical environment, Journal of Fluid Mechanics, 717 (2013) 193-202.

[42] A.A. Amsden, KIVA-3V, Release 2, Improvements to KIVA-3V, Los Alamos National Laboratory, 1999.

[43] R.D. Reitz, C.J. Rutland, Development and testing of diesel engine CFD models, Prog Energ Combust, 21(2) (1995) 173-196.

[44] J. Gustavsson, V.I. Golovitchev, Spray Combustion Simulation Based on Detailed Chemistry Approach for Diesel Fuel Surrogate Model, in, SAE International, 2003.

[45] A.A. Amsden, O'Rourke, P. J. and Butler, T. D., KIVA-II: a computer program for chemically reactive flows with sprays, Los Alamos National Laboratory, 1989.

[46] M.F. Trujillo, P.J. O'Rourke, D. Torres, Generalizing the Thermodynamics State Relationships in KIVA-3V, Los Alamos National Laboratories, 2002.

[47] M.F. Trujillo, D.J. Torres, P.J. O'Rourke, High-pressure multicomponent liquid sprays: Departure from ideal behaviour, International Journal of Engine Research, 5(3) (2004) 229-246.

[48] D.Y. Peng, D.B. Robinson, A New Two-Constant Equation of State, Ind Eng Chem Fund, 15(1) (1976) 59-64.

[49] X. Petit, G. Ribert, G. Lartigue, P. Domingo, Large-eddy simulation of supercritical fluid injection, The Journal of Supercritical Fluids, 84(0) (2013) 61-73.

[50] H.B. Callen, Thermodynamics and an introduction to thermostatics, 2nd ed., J. Wiley, New York ; Chichester etc., 1985.

[51] M. Castier, Solution of the isochoric-isoenergetic flash problem by direct entropy maximization, Fluid Phase Equilibr, 276(1) (2009) 7-17.

[52] L. Qiu, Y. Wang, R.D. Reitz, Multiphase dynamic flash simulations using entropy maximization and application to compressible flow with phase change, AIChE J, 60(8) (2014) 3013-3024.

[53] J.E. Dennis, R.B. Schnabel, Numerical methods for unconstrained optimization and nonlinear equations, Prentice-Hall, Englewood Cliffs, N.J., 1983.

[54] L. Qiu, Y. Wang, Q. Jiao, H. Wang, R.D. Reitz, Development of a thermodynamically consistent, robust and efficient phase equilibrium solver and its validations, Fuel, 115(0) (2014) 1-16.

[55] L. Qiu, Development of an isoenergetic-isochoric flash solver based on entropy maximization principle, Internal Research Report, University of Wisconsin-Madison, 2013, June.

[56] L. Qiu, Y. Wang, Q. Jiao, H. Wang, R.D. Reitz, Simulating condensation in a supercritical gas jet, in: ILASS Americas, 25th Annual Conference on Liquid Atomization and Spray Systems, Pittsburgh, PA, , May 5-8, 2013.

[57] L. Qiu, R.D. Reitz, Condensation processes in a motoring engine, The Journal of Supercritical Fluids, 90(0) (2014) 84-100.

[58] M. Castier, Dynamic simulation of fluids in vessels via entropy maximization, J Ind Eng Chem, 16(1) (2010) 122-129.

[59] M.L. Michelsen, The Isothermal Flash Problem .1. Stability, Fluid Phase Equilibr, 9(1) (1982) 1-19.

[60] K.E.T. Giljarhus, S.T. Munkejord, G. Skaugen, Solution of the Span-Wagner Equation of State Using a Density-Energy State Function for Fluid-Dynamic Simulation of Carbon Dioxide, Ind Eng Chem Res, 51(2) (2012) 1006-1014.

[61] A. Firoozabadi, Thermodynamics of hydrocarbon reservoirs, McGraw-Hill, New York, 1999.

[62] S.I. Sandler, Chemical and engineering thermodynamics, 3rd ed., Wiley, New York, 1999. 
[63] C.K. Muthukumaran, A. Vaidyanathan, Experimental study of elliptical jet from sub to supercritical conditions, Physics of Fluids (1994-present), 26(4) (2014) -.

[64] P.E. Tuma, Fluoroketone $\mathrm{C} 2 \mathrm{~F} 5 \mathrm{C}(\mathrm{O}) \mathrm{CF}(\mathrm{CF} 3) 2$ as a Heat Transfer Fluid for Passive and Pumped 2-Phase Applications, in: Semiconductor Thermal Measurement and Management Symposium, 2008. Semi-Therm 2008. Twenty-fourth Annual IEEE, 2008, pp. 173-179.

[65] R.L. Rowley, W.V. Wilding, J.L. Oscarson, N. Giles, R.J. Rowley, T.E. Daubert, R.P. Danner, DIPPR Data Compilation of Pure Compound Properties, in, 2001.

[66] R.C. Reid, J.M. Prausnitz, B.E. Poling, The properties of gases and liquids, 4th ed., McGraw-Hill, New York, 1987.

[67] S. Gordon, B.J. McBride, Computer Program for Calculation of Complex Chemical Equilibrium Compositions and Applications: I. Analysis, NASA Reference Publication 1311, (October 1994).

[68] K. Tochigi, T. Namae, T. Suga, H. Matsuda, K. Kurihara, M.C. dos Ramos, C. McCabe, Measurement and prediction of high-pressure vapor-liquid equilibria for binary mixtures of carbon dioxide+n-octane, methanol, ethanol, and perfluorohexane, The Journal of Supercritical Fluids, 55(2) (2010) 682-689.

[69] P.J. Linstrom, W.G. Mallard, NIST Chemistry WebBook, NIST Standard Reference Database Number 69, in, National Institute of Standards and Technology, Gaithersburg MD.

[70] F. Garcia-Sanchez, G. Eliosa-Jimenez, G. Silva-Oliver, A. Godinez-Silva, High-pressure (vapor plus liquid) equilibria in the (nitrogen plus n-heptane) system, J Chem Thermodyn, 39(6) (2007) 893-905.

[71] G. Eliosa-Jiménez, F. García-Sánchez, G. Silva-Oliver, R. Macías-Salinas, Vapor-liquid equilibrium data for the nitrogen+n-octane system from (344.5 to 543.5) $\mathrm{K}$ and at pressures up to $50 \mathrm{MPa}$, Fluid Phase Equilibr, 282(1) (2009) 3-10.

[72] H.Q. Pan, Firoozabadi, A., Two-phase isentropic compressibility and two-phase sonic velocity for multicomponent-hydrocarbon mixtures, SPE Reserv Eval Eng, 3(4) (2000) 335-341.

[73] D.V. Nichita, P. Khalid, D. Broseta, Calculation of isentropic compressibility and sound velocity in two-phase fluids, Fluid Phase Equilibr, 291(1) (2010) 95-102.

[74] M. Castier, Thermodynamic speed of sound in multiphase systems, Fluid Phase Equilibr, 306(2) (2011) 204-211.

[75] B. Chehroudi, D. Talley, E. Coy, Visual characteristics and initial growth rates of round cryogenic jets at subcritical and supercritical pressures, Physics of Fluids, 14(2) (2002) 850-861.

[76] A. Roy, C. Segal, C. Joly, Spreading Angle and Core Length Analysis of Supercritical Jets, Aiaa J, 51(8) (2013) 2009-2014.

[77] D.B. Robinson, D.Y. Peng, The characterization of the heptanes and heavier fractions for the GPA Peng-Robinson programs, Research report RR-28, Gas Processors Association, 1978.

[78] P.G. Debenedetti, Homogeneous nucleation in supercritical fluids, AIChE J, 36(9) (1990) 1289-1298.

[79] F.F. Abraham, Homogeneous nucleation theory the pretransition theory of vapor condensation, in: Advances in theoretical chemistry. Supplement 1, Academic Press, New York,, 1974. 
Table 1. Thermodynamic properties of pure species.

\begin{tabular}{|c|c|c|c|c|}
\hline species & $\begin{array}{c}\boldsymbol{T}_{\boldsymbol{c}} \\
(\mathbf{K})\end{array}$ & $\begin{array}{c}\boldsymbol{P}_{\boldsymbol{c}} \\
(\mathbf{b a r})\end{array}$ & $\begin{array}{c}\boldsymbol{\omega} \\
(-)\end{array}$ & $\begin{array}{c}\boldsymbol{M W} \\
(\mathbf{g} / \mathbf{m o l e})\end{array}$ \\
\hline $\mathrm{N}_{2}$ & 126.2 & 33.9 & 0.0377 & 28.0 \\
\hline $\mathrm{CO}_{2}$ & 304.21 & 73.83 & 0.2236 & 44.0 \\
\hline $\mathrm{C}_{6} \mathrm{~F}_{14}$ & 449.55 & 18.02 & 0.49653 & 338.0 \\
\hline $\mathrm{C}_{6} \mathrm{~F}_{12} \mathrm{O}$ & 442.0 & 18.7 & 0.6274 & 316.0 \\
\hline
\end{tabular}

Table 2. Simulation conditions for supercritical injection.

\begin{tabular}{|c|c|c|c|c|c|c|c|}
\hline \multirow{2}{*}{ Case } & \multicolumn{2}{|c|}{ Chamber conditions } & \multicolumn{7}{c|}{ Injection conditions } \\
\cline { 2 - 8 } & $\begin{array}{c}\text { Reduced } \\
\text { temperature }\end{array}$ & $\begin{array}{c}\text { Reduced } \\
\text { pressure }\end{array}$ & $\begin{array}{c}\text { Reduced } \\
\text { temperature }\end{array}$ & $\begin{array}{c}\text { Reduced } \\
\text { pressure }\end{array}$ & $\begin{array}{c}\text { velocity } \\
(\mathrm{m} / \mathrm{s})\end{array}$ & $\mathrm{Ma}$ & $\mathrm{Re}$ \\
\hline 1 & 0.69 & 1.38 & 1.13 & 1.51 & 20.98 & 0.27 & $8.6 \mathrm{e}+05$ \\
\hline 2 & 0.72 & 1.86 & 1.21 & 1.97 & 17.95 & 0.19 & $7.6 \mathrm{e}+05$ \\
\hline 3 & 0.76 & 1.88 & 1.29 & 1.98 & 20.31 & 0.20 & $6.8 \mathrm{e}+05$ \\
\hline 4 & 0.80 & 1.38 & 1.31 & 1.51 & 30.00 & 0.28 & $7.0 \mathrm{e}+05$ \\
\hline 5 & 1.04 & 1.26 & 1.00 & 1.34 & 7.07 & 0.08 & $8.3 \mathrm{e}+05$ \\
\hline 6 & 1.06 & 1.37 & 1.08 & 1.47 & 14.56 & 0.22 & $7.8 \mathrm{e}+05$ \\
\hline 7 & 1.08 & 1.41 & 1.08 & 1.5 & 14.47 & 0.22 & $8.1 \mathrm{e}+05$ \\
\hline 8 & 1.09 & 1.47 & 1.18 & 1.67 & 20.57 & 0.24 & $7.9 \mathrm{e}+05$ \\
\hline 9 & 0.8 & 1.38 & 1.13 & 1.51 & 20.98 & 0.27 & $8.6 \mathrm{e}+05$ \\
\hline 10 & 0.6 & 1.38 & 1.13 & 1.51 & 20.98 & 0.27 & $8.6 \mathrm{e}+05$ \\
\hline
\end{tabular}

Table B1. Parameters and calculated characteristic time scales required for phase equilibrium

\begin{tabular}{|c|c|c|c|c|c|}
\hline $\begin{array}{c}\boldsymbol{T} \\
(\mathbf{K})\end{array}$ & $\begin{array}{c}\boldsymbol{P} \\
(\mathbf{b a r})\end{array}$ & $\begin{array}{c}\boldsymbol{x}^{\text {liq }} \\
(-)\end{array}$ & $\begin{array}{c}\boldsymbol{\rho}^{\text {liq,sat }} \\
\left(\mathbf{k m o l e} / \mathbf{m}^{\mathbf{3}}\right)\end{array}$ & $\begin{array}{c}\boldsymbol{\sigma} \\
(\mathbf{N} / \mathbf{m})\end{array}$ & $\begin{array}{c}\boldsymbol{\tau} \\
(\mathbf{s})\end{array}$ \\
\hline 250 & 20 & $1.0 \mathrm{e}-03$ & 5.3697 & 0.01572 & $4.2 \mathrm{e}-08$ \\
\hline 250 & 30 & $5.0 \mathrm{e}-03$ & 5.3697 & 0.01572 & $5.6 \mathrm{e}-09$ \\
\hline 300 & 20 & $2.0 \mathrm{e}-03$ & 5.0031 & 0.01111 & $2.8 \mathrm{e}-08$ \\
\hline 300 & 30 & $4.0 \mathrm{e}-03$ & 5.0031 & 0.01111 & $9.3 \mathrm{e}-09$ \\
\hline 350 & 20 & $1.0 \mathrm{e}-03$ & 4.4838 & 0.0068 & $7.5 \mathrm{e}-08$ \\
\hline 350 & 30 & $1.0 \mathrm{e}-03$ & 4.4838 & 0.0068 & $5.0 \mathrm{e}-08$ \\
\hline
\end{tabular}




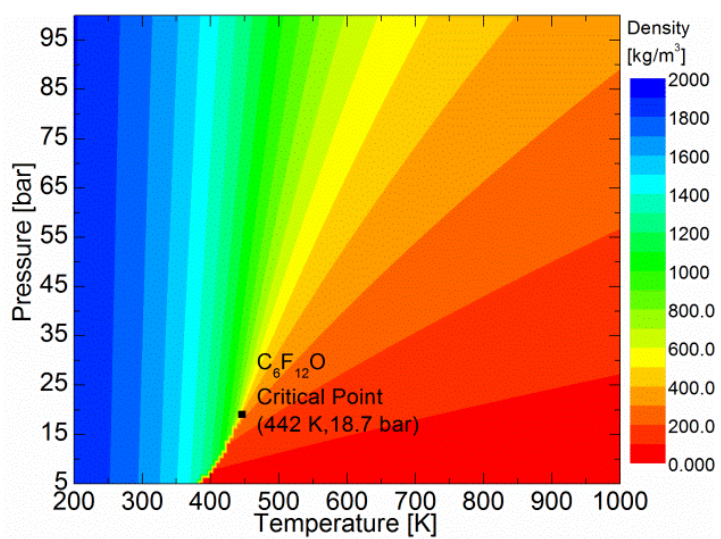

(a)

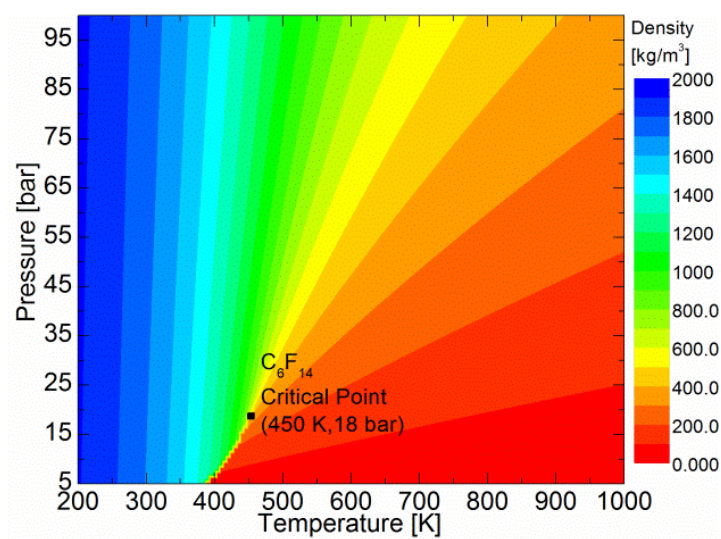

(b)

Fig. 1. Density contour of pure species on the pressure-temperature diagram using the PengRobinson equation of state model. (a) Fluoroketone $\left(\mathrm{C}_{6} \mathrm{~F}_{12} \mathrm{O}\right)$. (b) Perfluorohexane $\left(\mathrm{C}_{6} \mathrm{~F}_{14}\right)$.

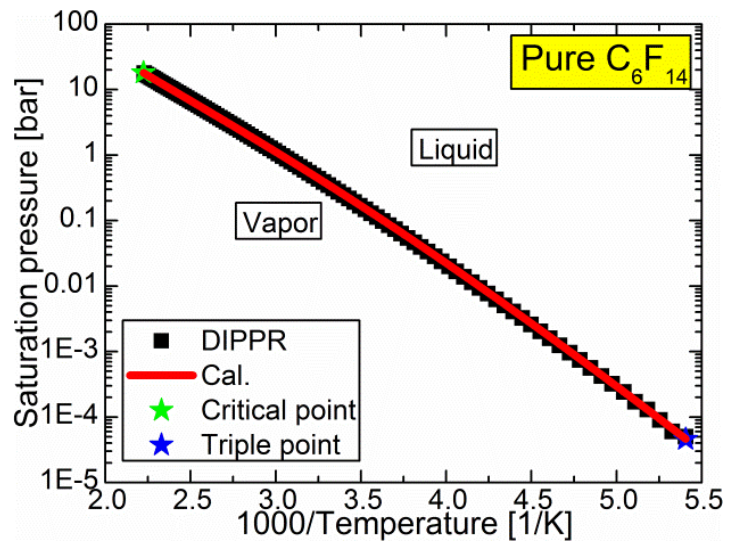

(a)

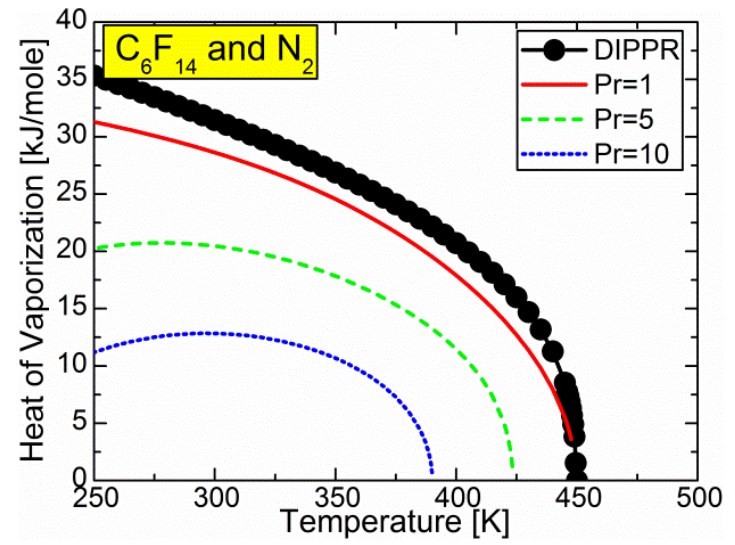

(b)

Fig. 2. (a) $\log (\mathrm{P})$ vs. $1000 / \mathrm{T}$ relationship for pure $\mathrm{C}_{6} \mathrm{~F}_{14}$ from the triple to the critical points. (b) Pressure effects on the heat of vaporization of $\mathrm{C}_{6} \mathrm{~F}_{14}$ for a binary mixture. 


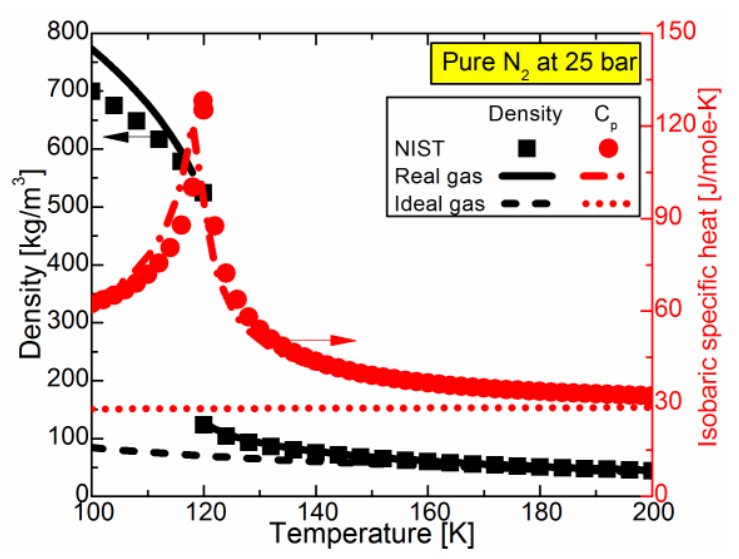

(a)

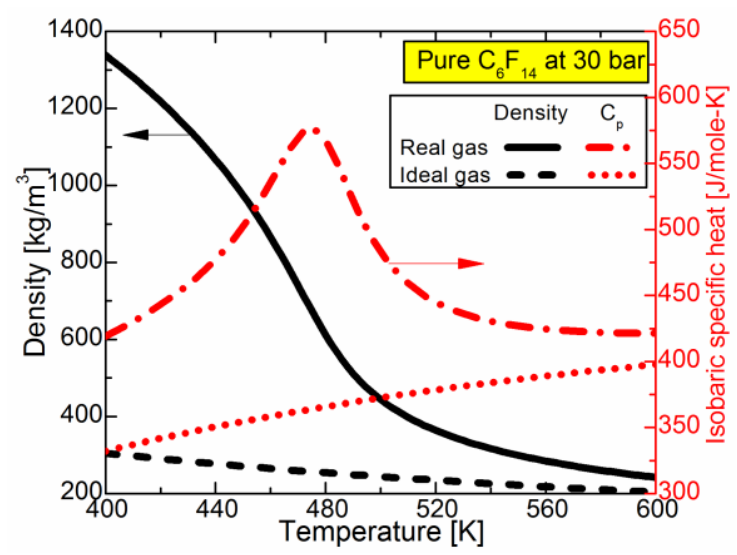

(b)

Fig. 3. Predicted density and isobaric specific heat capacity with available NIST data. (a) Pure nitrogen at 25 bar. (b) Pure $\mathrm{C}_{6} \mathrm{~F}_{14}$ at 30 bar.

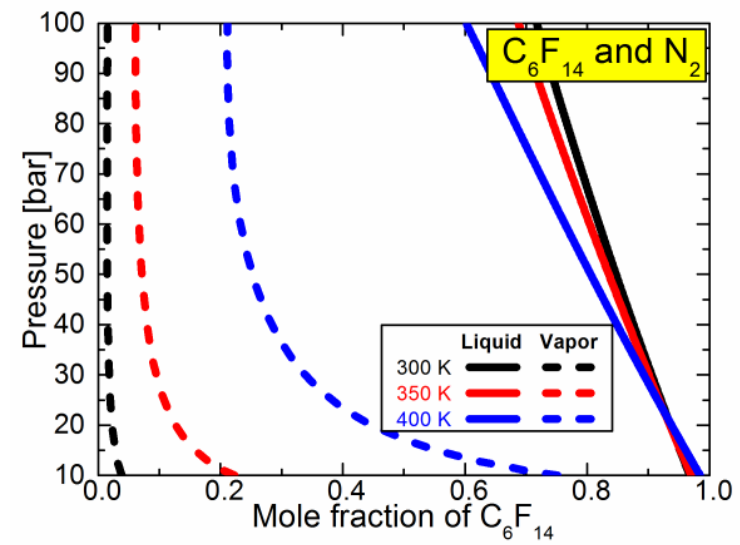

(a)

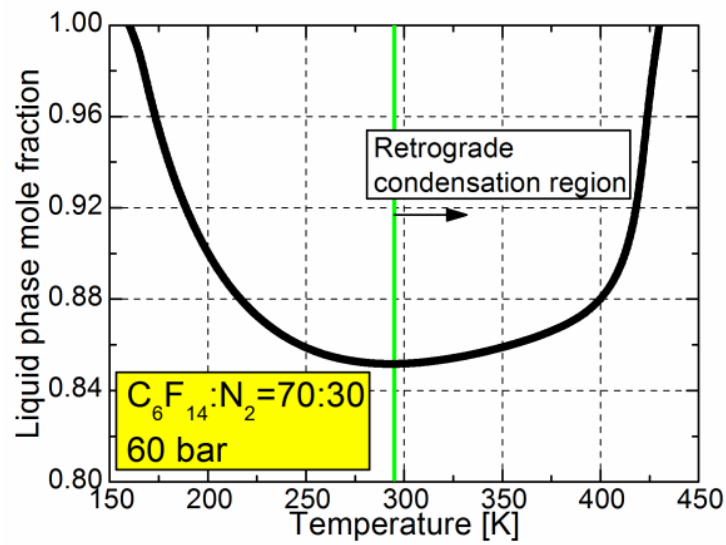

(b)

Fig. 4. Phase behavior of binary $\mathrm{C}_{6} \mathrm{~F}_{14}$ and $\mathrm{N}_{2}$ mixtures. (a) Pressure-mole fraction phase diagram at three temperatures. (b) Retrograde condensation behavior at 60 bar for the 70:30 (mole) mixture. 


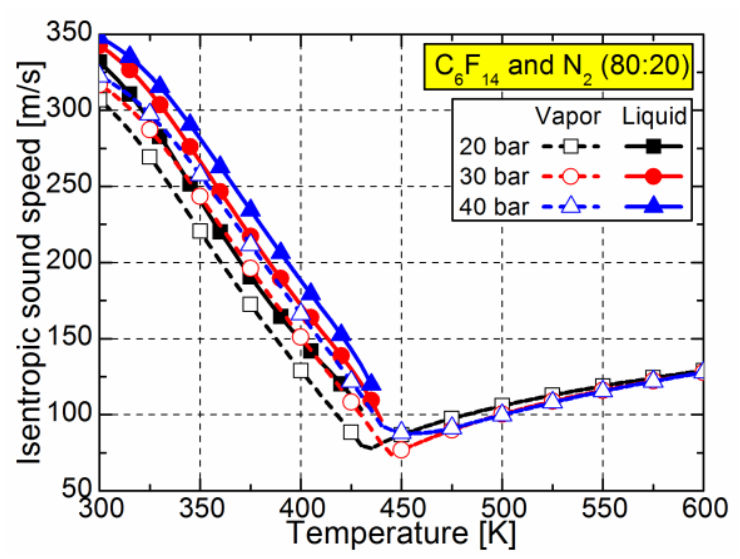

(a)

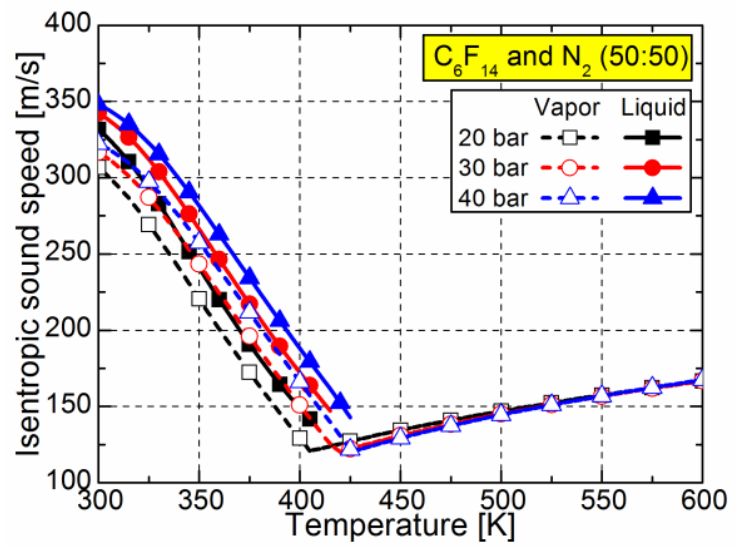

(c)

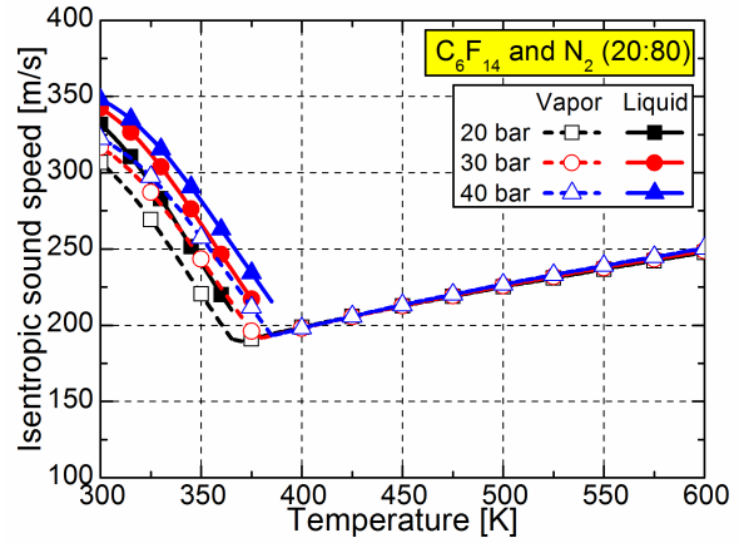

(e)

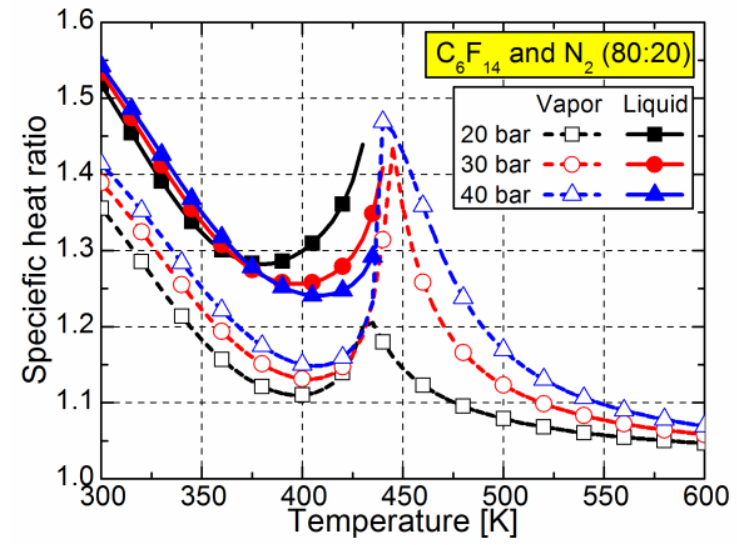

(b)

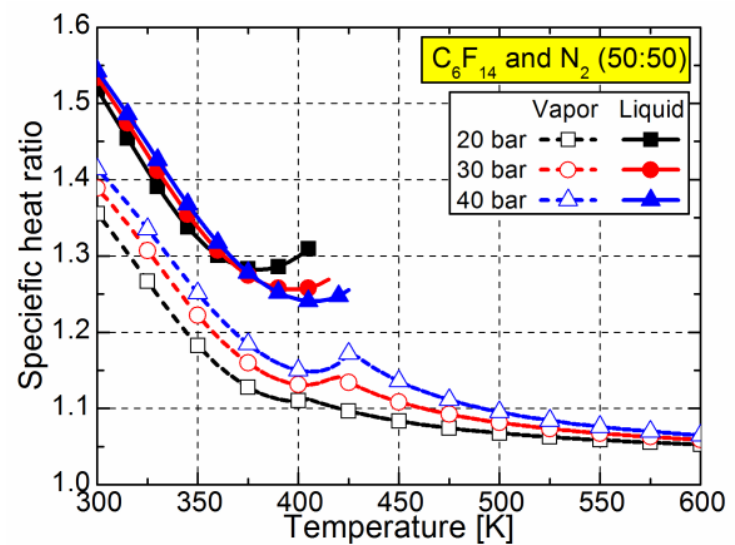

(d)

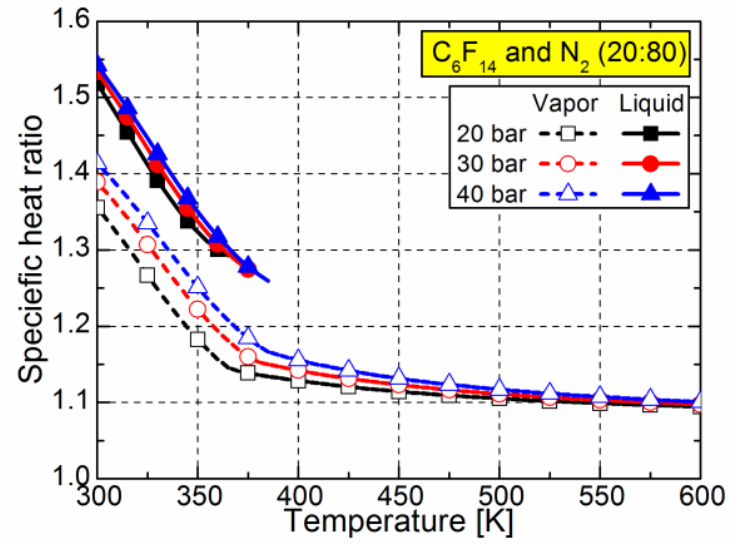

(f)

Fig. 5. Calculated thermodynamic properties for $\mathrm{C}_{6} \mathrm{~F}_{14}-\mathrm{N}_{2}$ mixtures at three compositions (in mole). (a), (c) and (e) Isentropic sound speed. (b), (d) and (f) Specific heat ratio. 


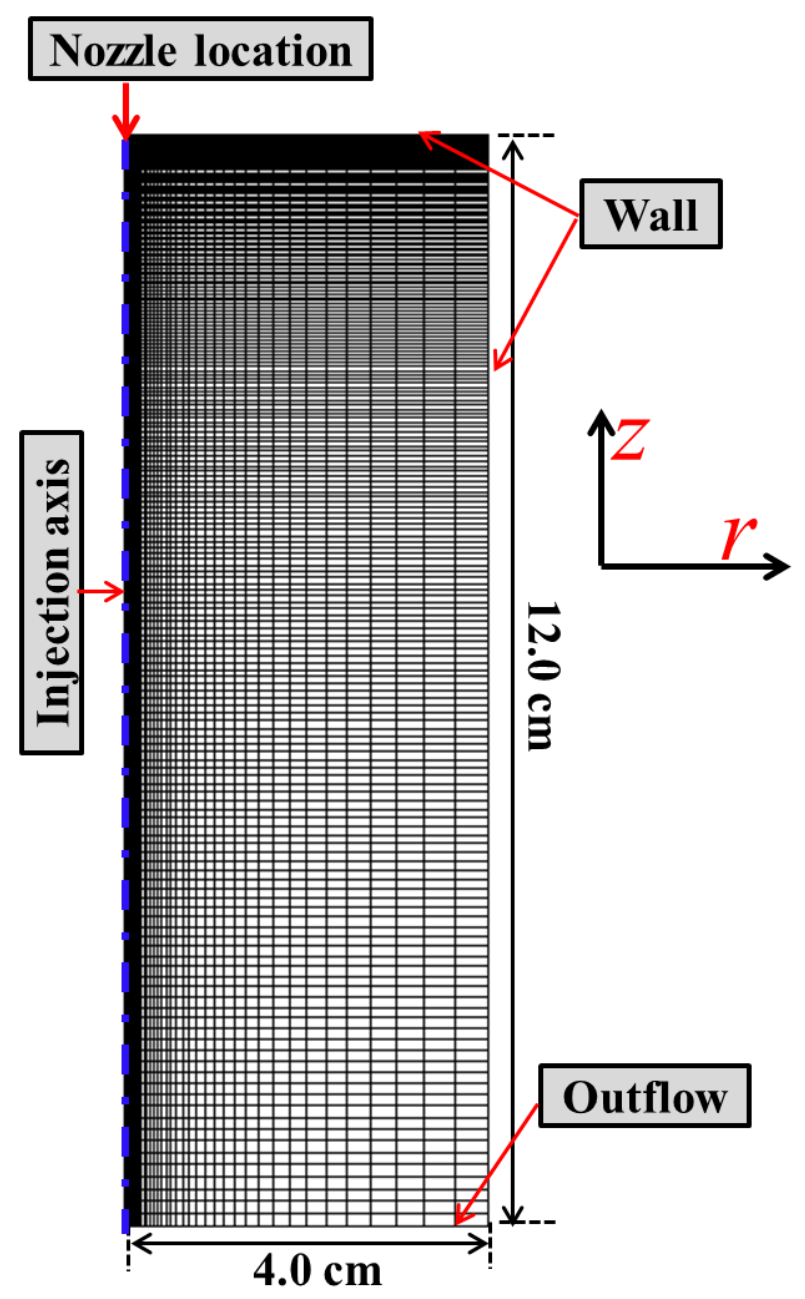

Fig. 6. 2-D axisymmetric computational mesh with radially (in $\mathrm{r}$ direction) and axially (in $\mathrm{z}$ direction) increasing grid spacing. Blue dash dot line shows the injection axis. Injector is located at the top. Outlet flow is at the bottom. All walls are adiabatic. 


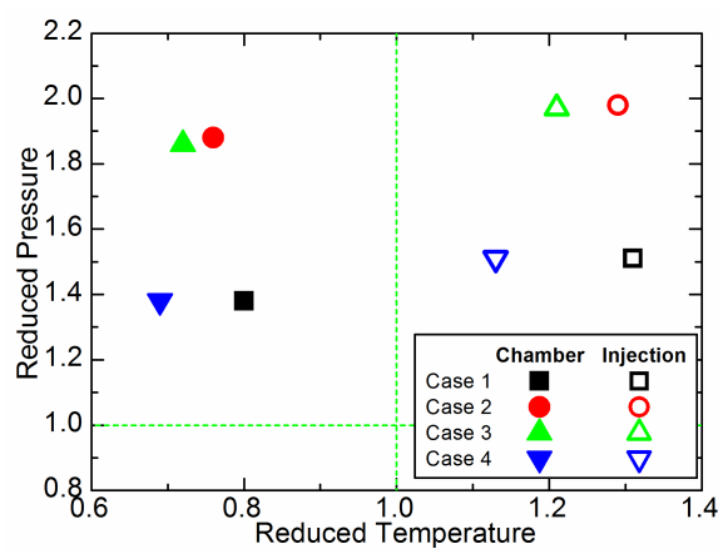

(a)

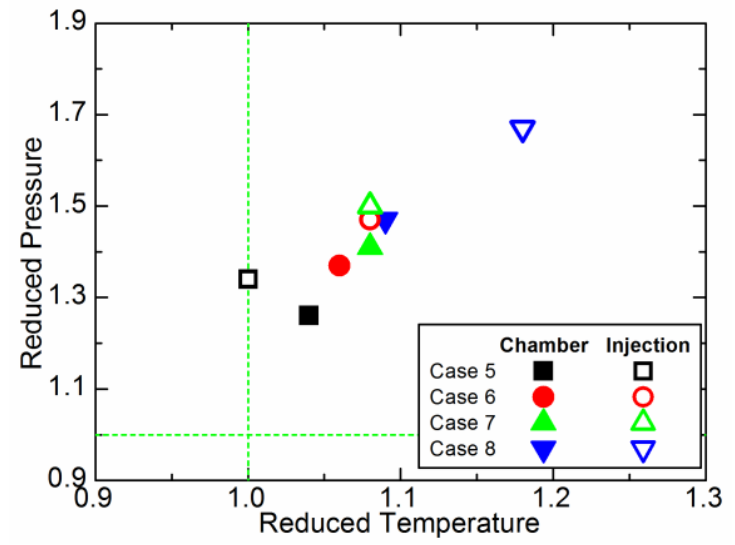

(b)

Fig. 7. Experimental conditions. (a) Cases 1-4 are supercritical-to-subcritical injections. (b) Cases 5-8 are supercritical-to-supercritical injections. Vertical dashed lines represent critical temperature and pressure of pure $\mathrm{C}_{6} \mathrm{~F}_{14}$. Refer to Table 2 for experimental conditions. 


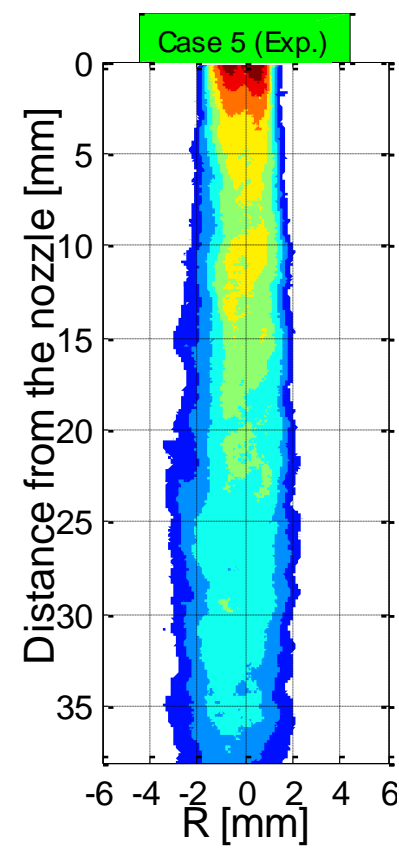

(a)

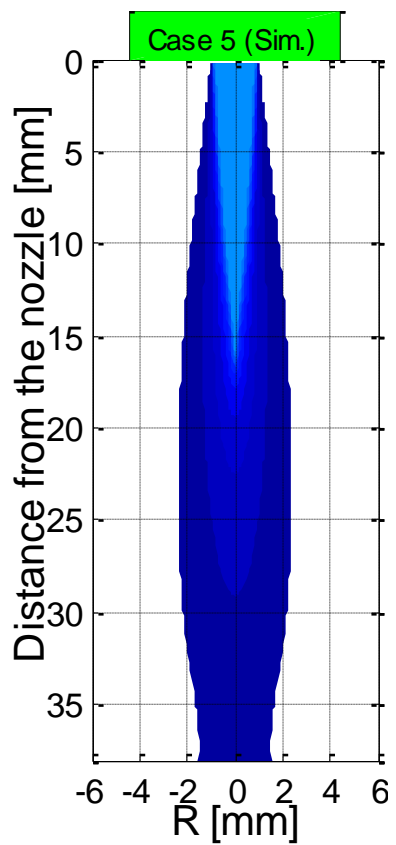

(b)

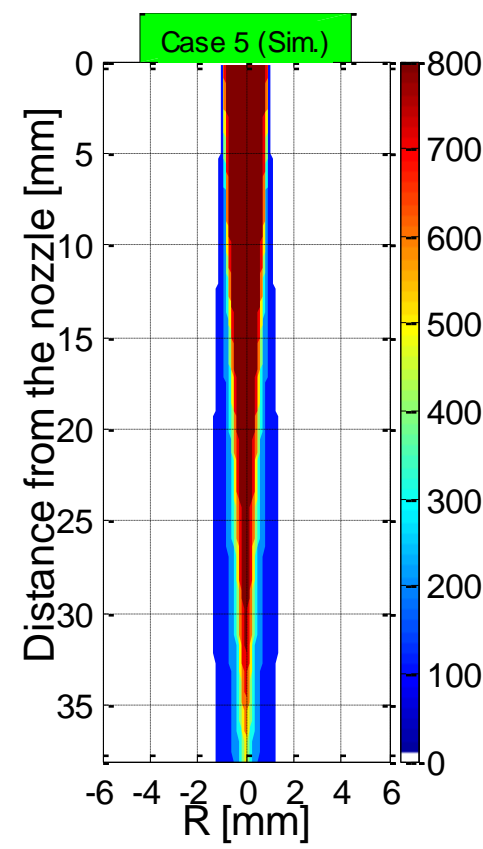

(c)

Fig. 8. Injectant density for Case 5. (a) Experimental data (b) Ideal gas simulation. (c) Real gas simulation.

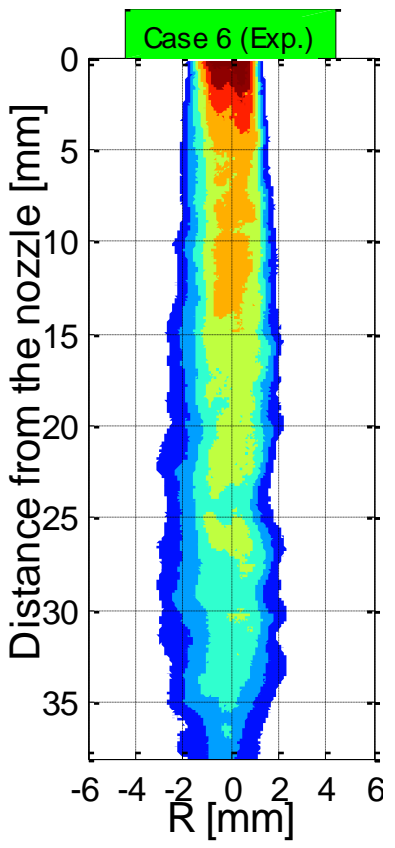

(a)

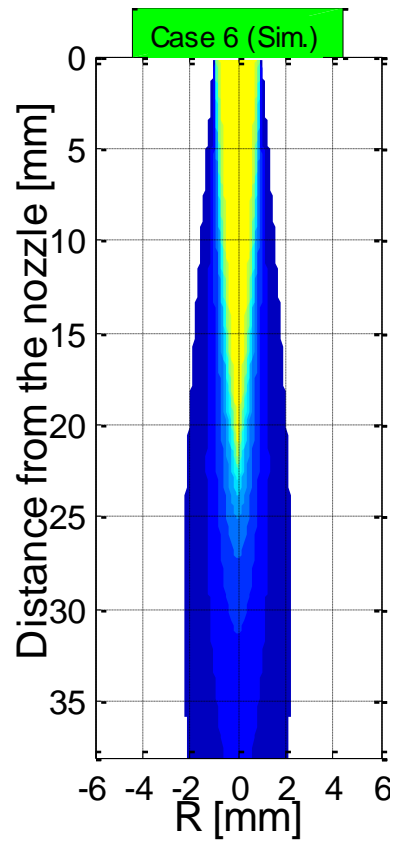

(b)

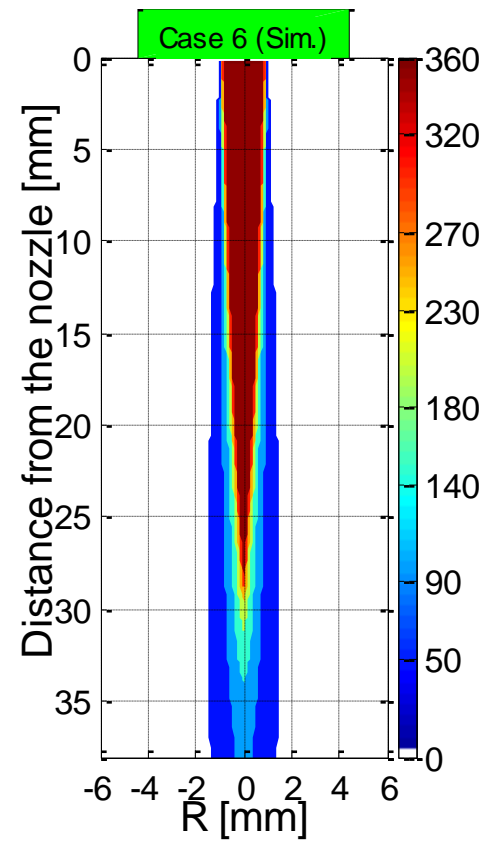

(c)

Fig. 9. Injectant density for Case 6. (a) Experimental data (b) Ideal gas simulation. (c) Real gas simulation. 


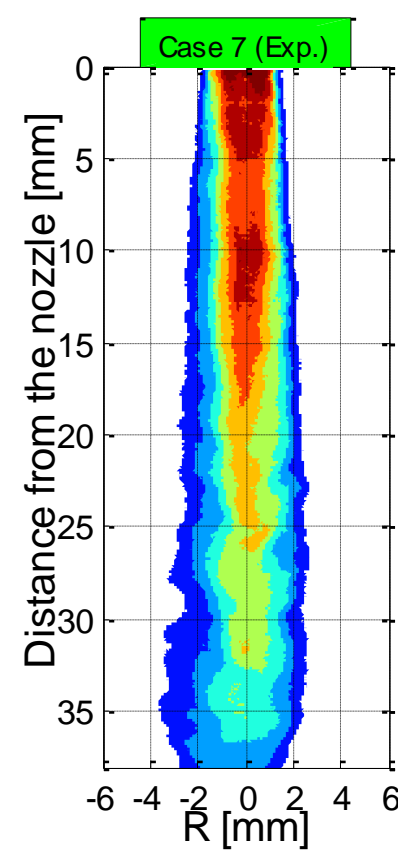

(a)

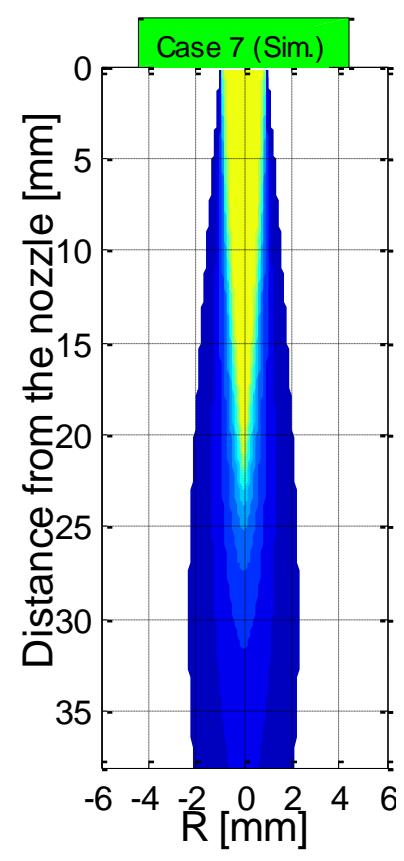

(b)

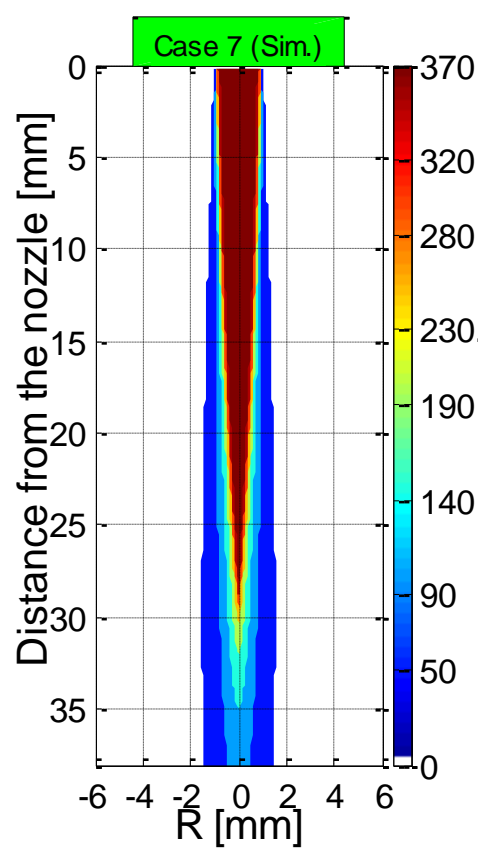

(c)

Fig. 10. Injectant density for Case 7. (a) Experimental data (b) Ideal gas simulation. (c) Real gas simulation.

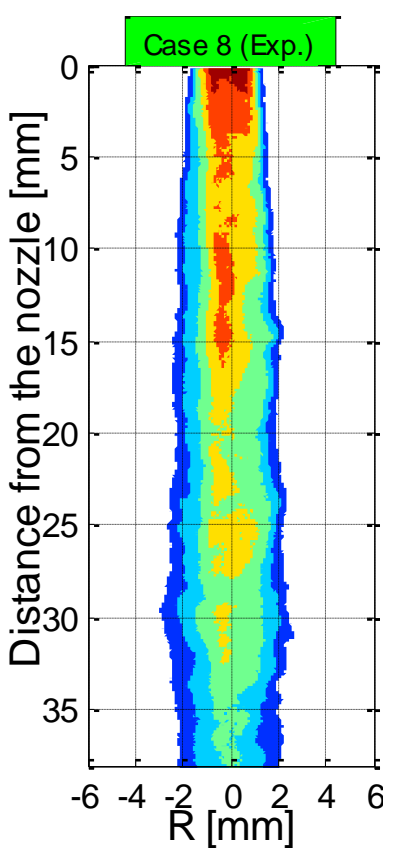

(a)

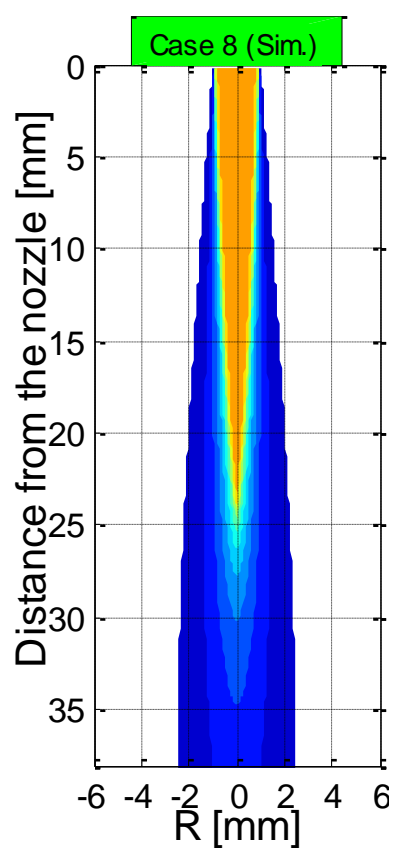

(b)

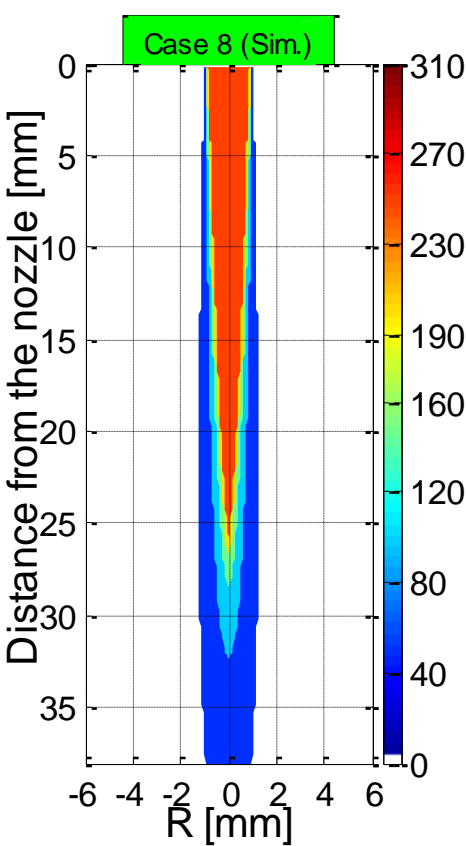

(c)

Fig. 11. Injectant density for Case 8. (a) Experimental data (b) Ideal gas simulation. (c) Real gas simulation. 


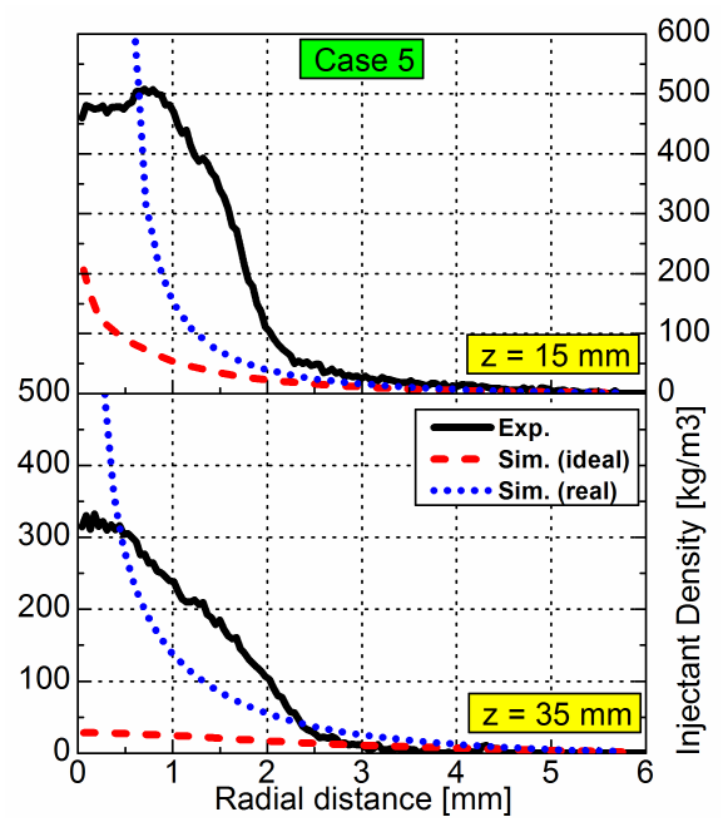

(a)

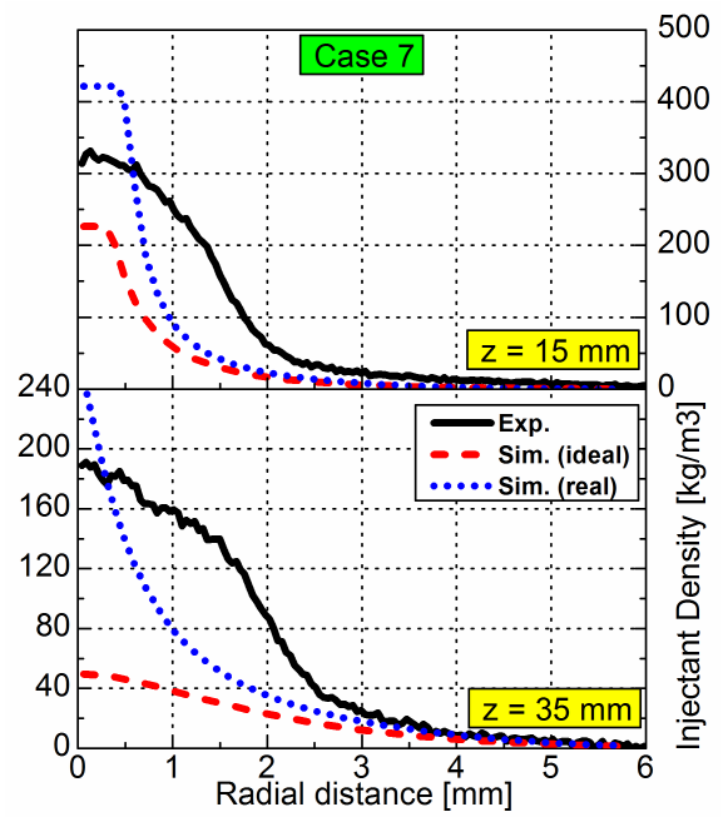

(c)

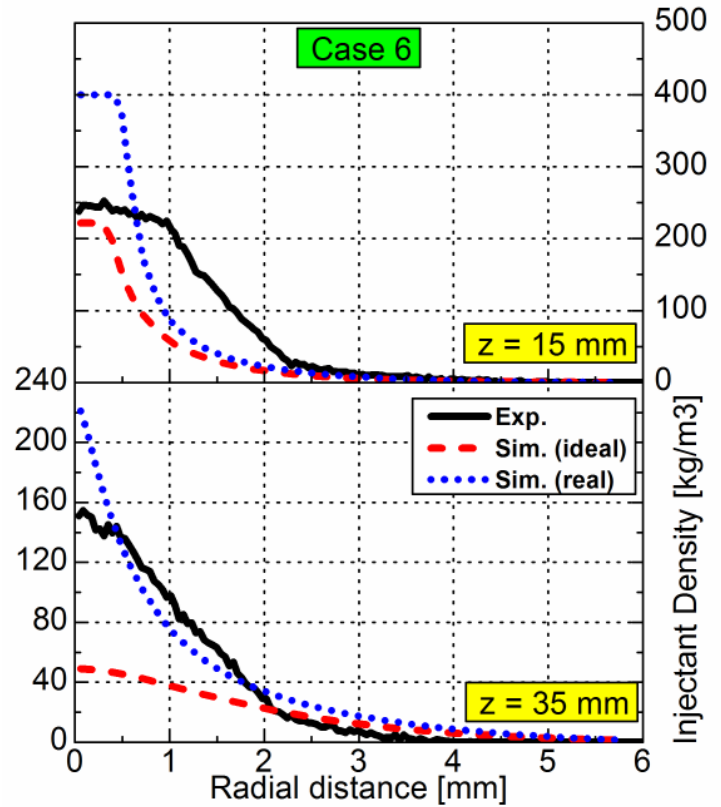

(b)

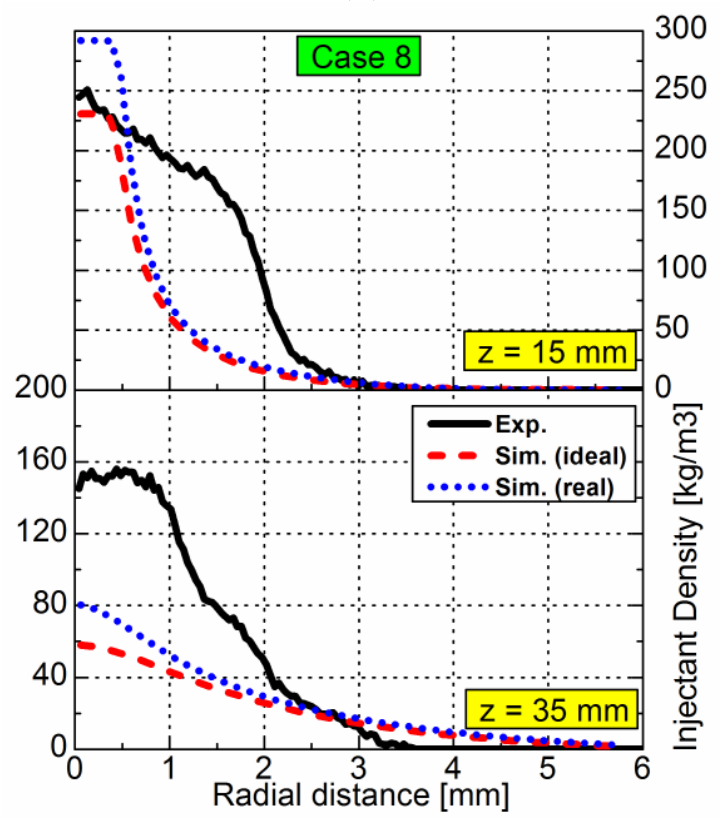

(d)

Fig. 12. Radial injectant density profiles at 15 and $35 \mathrm{~mm}$ downstream of the nozzle exit. (a) Case 5. (b) Case 6. (c) Case 7. (d) Case 8. 


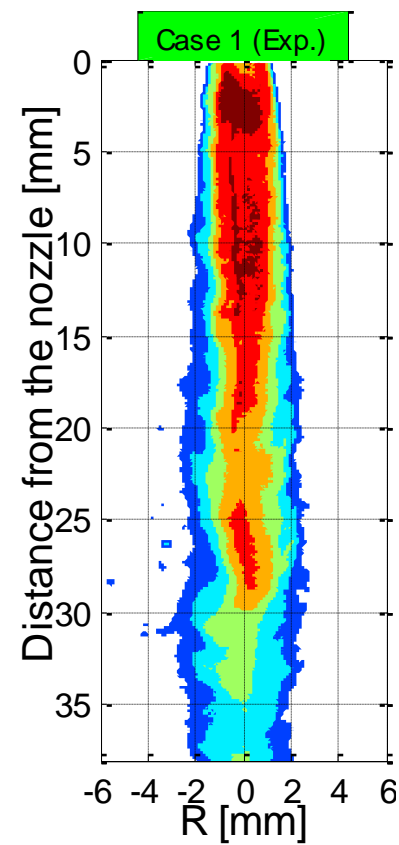

(a)

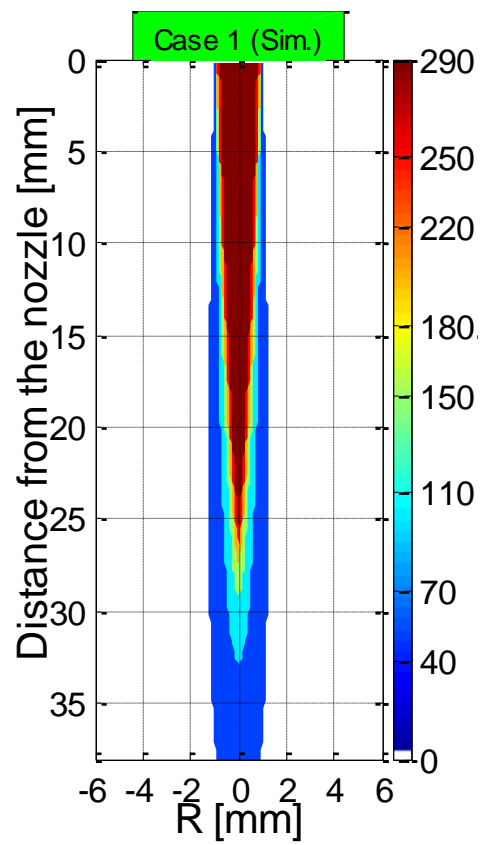

(b)

Fig. 13. Injectant density for Case 1. (a) Experiments. (b) Real gas simulation.

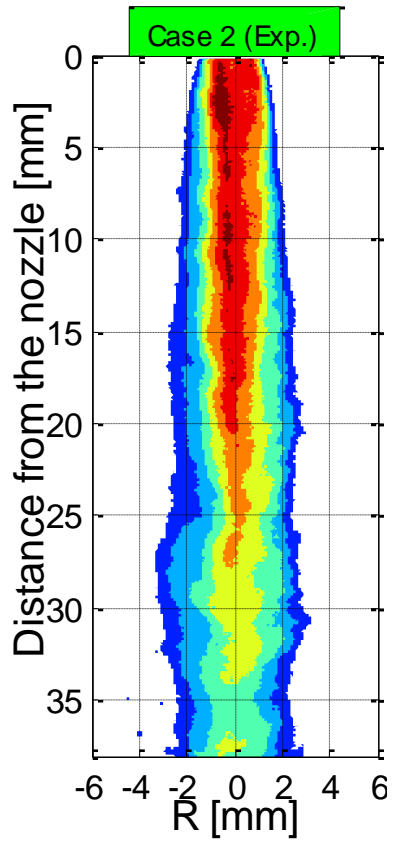

(a)

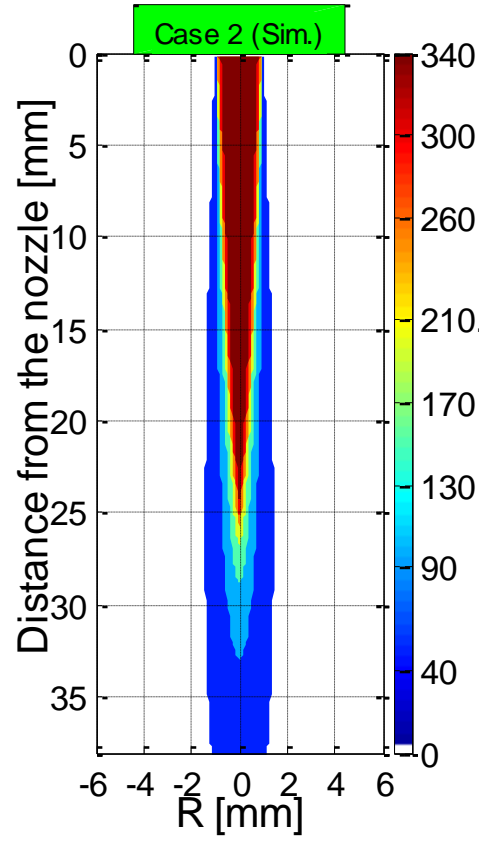

(b)

Fig. 14. Injectant density for Case 2. (a) Experiments. (b) Real gas simulation. 


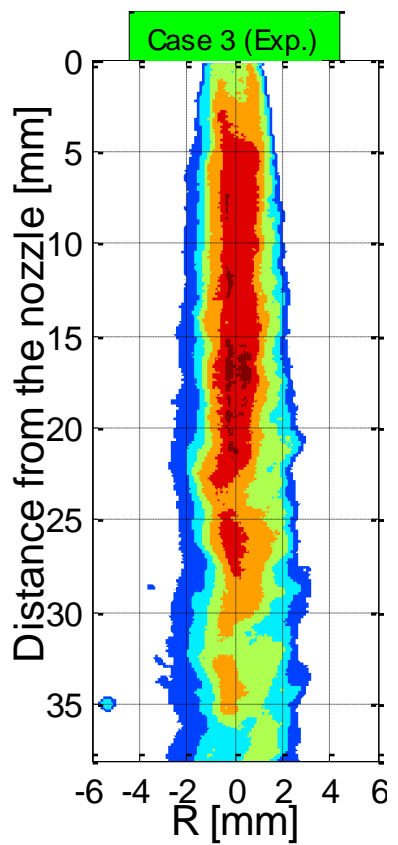

(a)

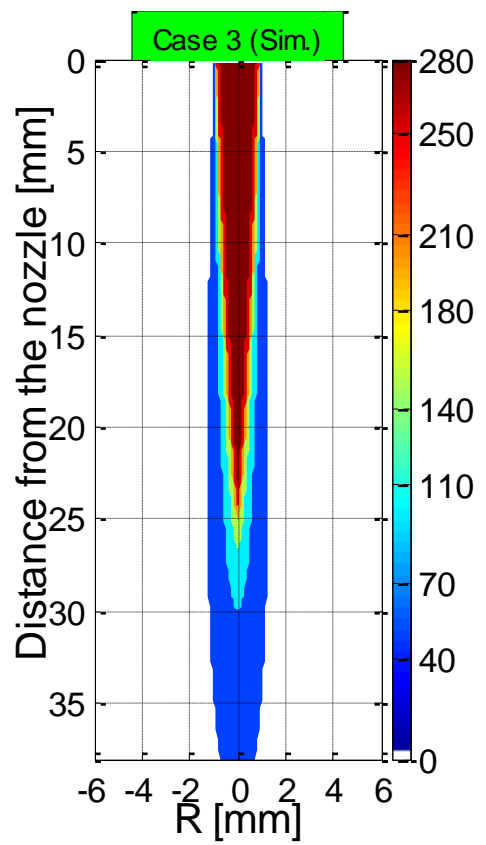

(b)

Fig. 15. Injectant density for Case 3. (a) Experiments. (b) Real gas simulation.

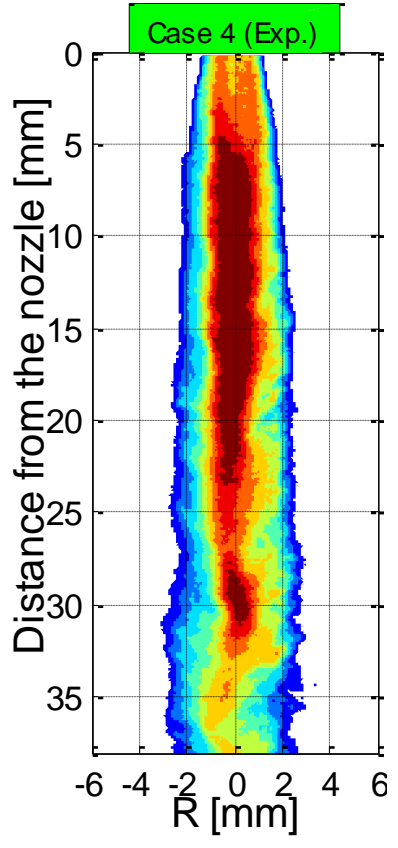

(a)

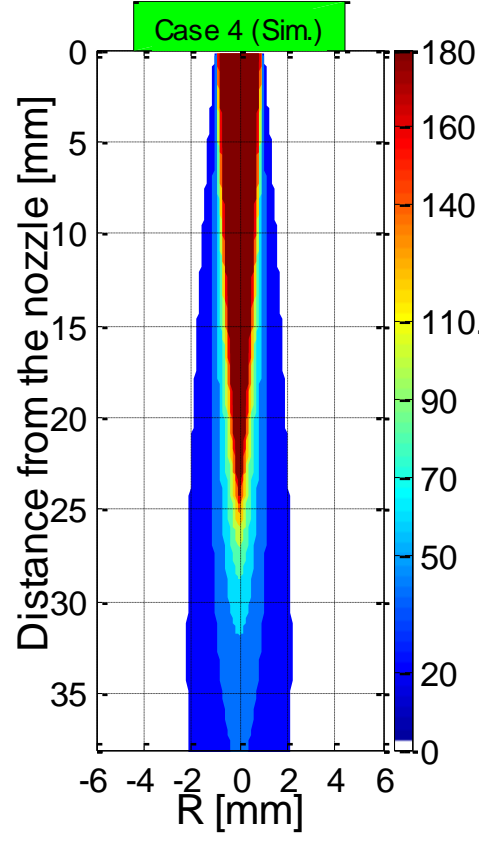

(b)

Fig. 16. Injectant density for Case 4. (a) Experiments. (b) Real gas simulation. 


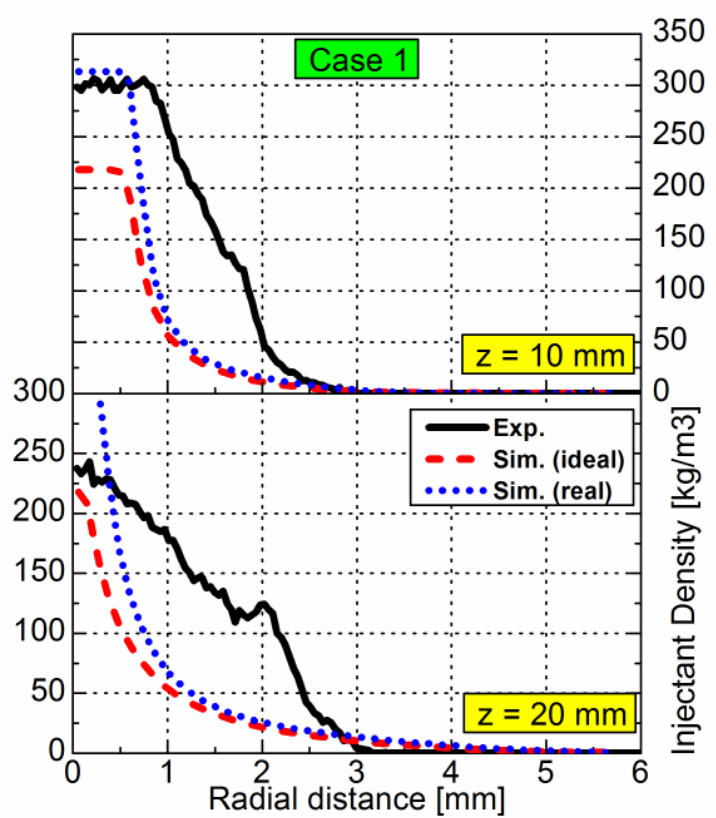

(a)

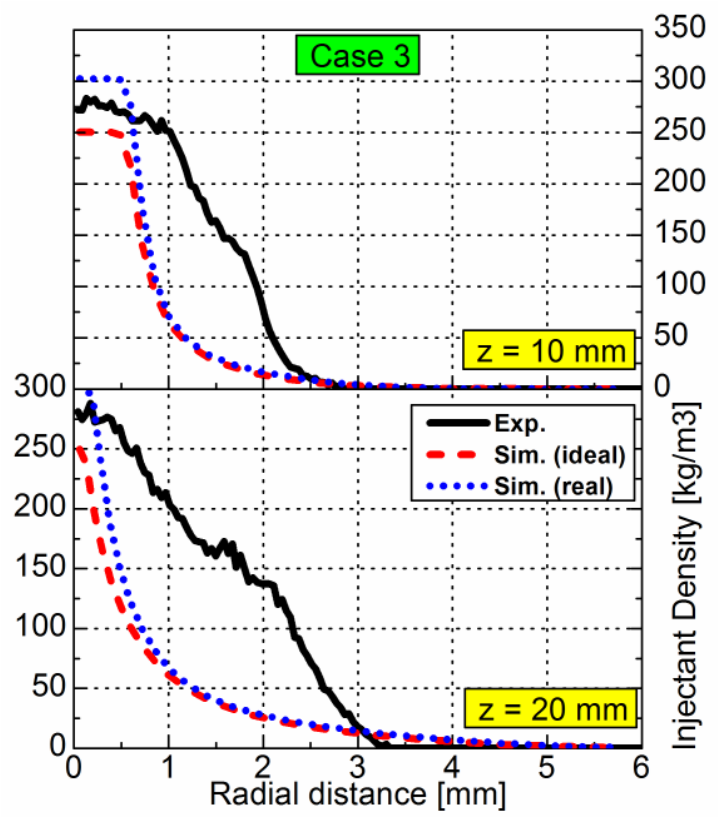

(d)

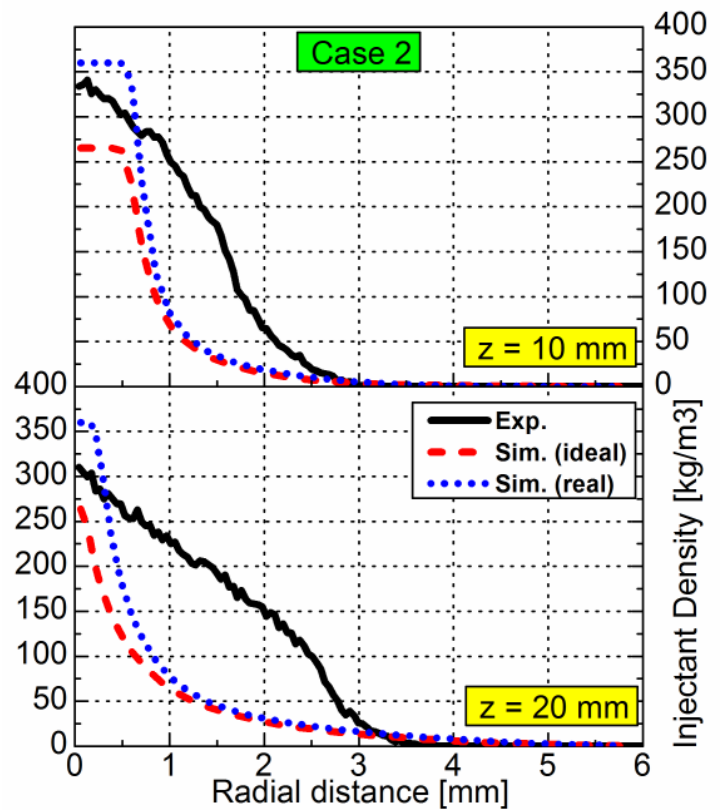

(b)

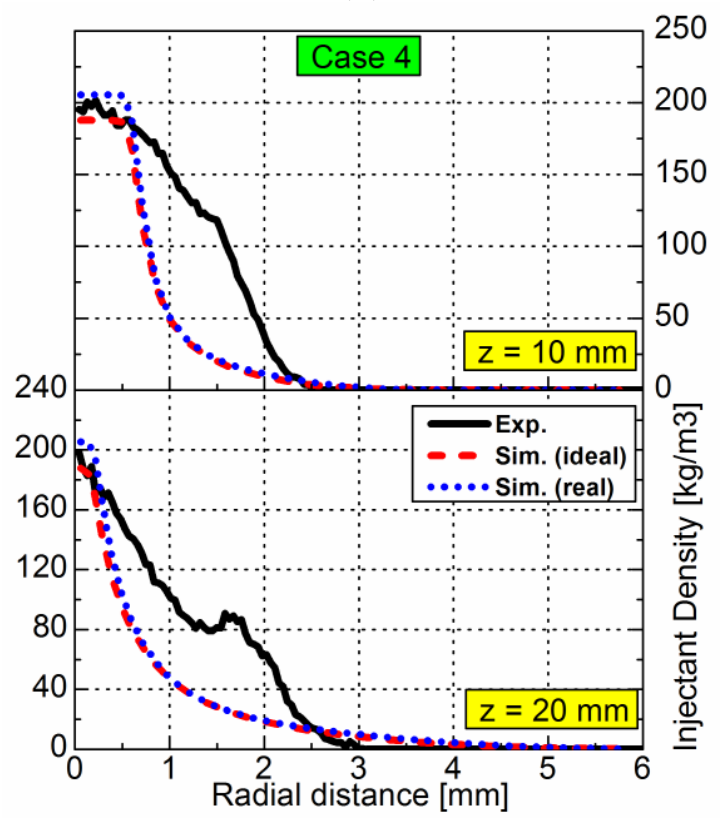

(d)

Fig. 17. Radial injectant density profiles at 10 and $20 \mathrm{~mm}$ downstream of the nozzle exit. (a) Case 1. (b) Case 2. (c) Case 3. (d) Case 4. 


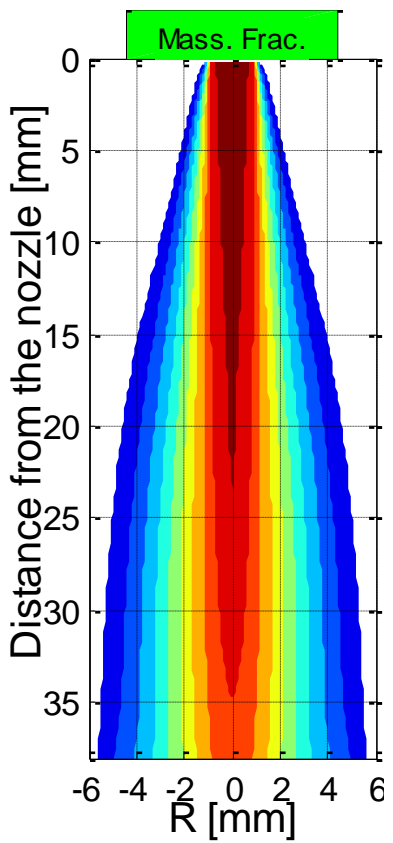

(a)

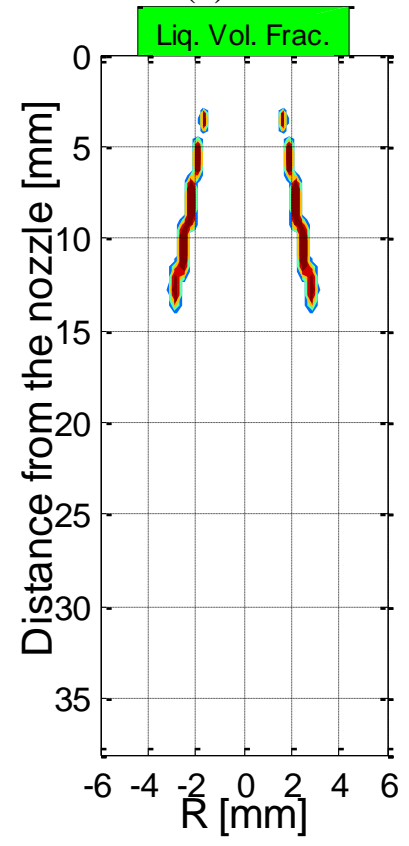

(d)

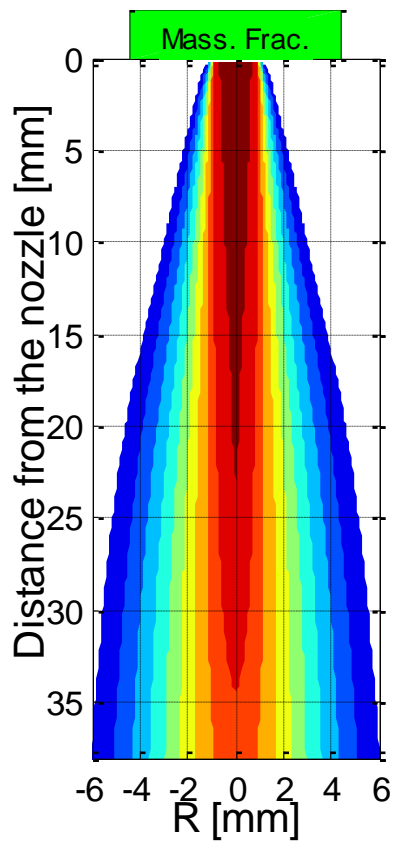

(b)

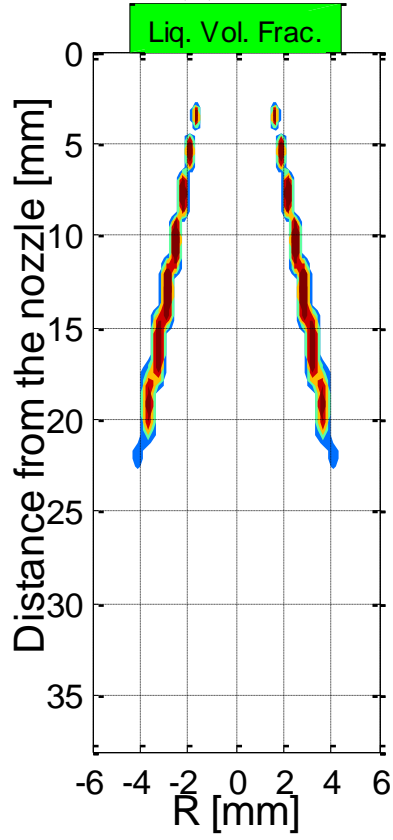

(e)

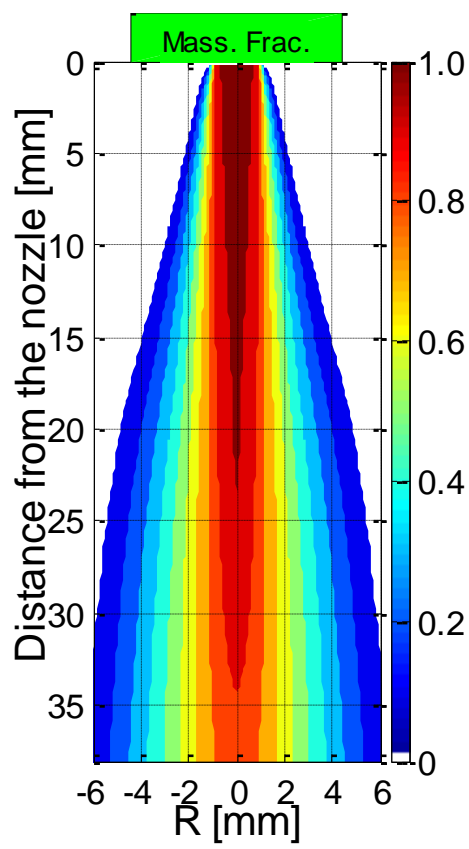

(c)

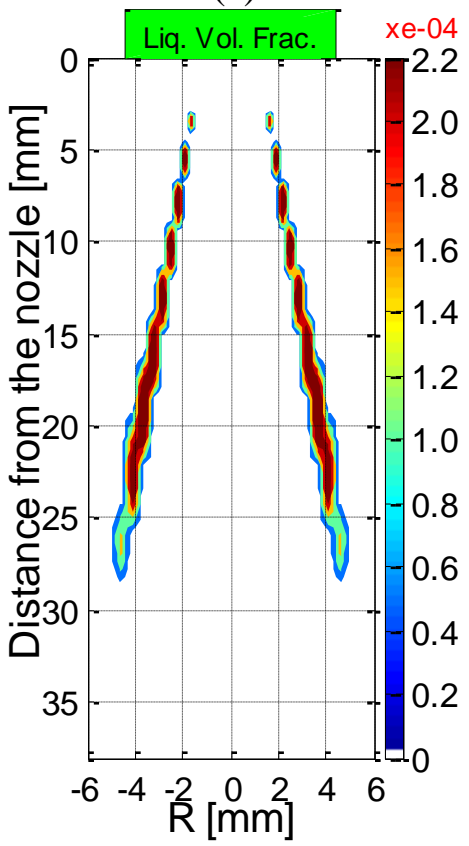

(f)

Fig. 18. Evolution of turbulent kinetic energy and liquid volume fraction at three time instants for Case 1. (a) and (d) 3ms ASI. (b) and (e) 5ms ASI. (c) and (f) 9ms ASI. 

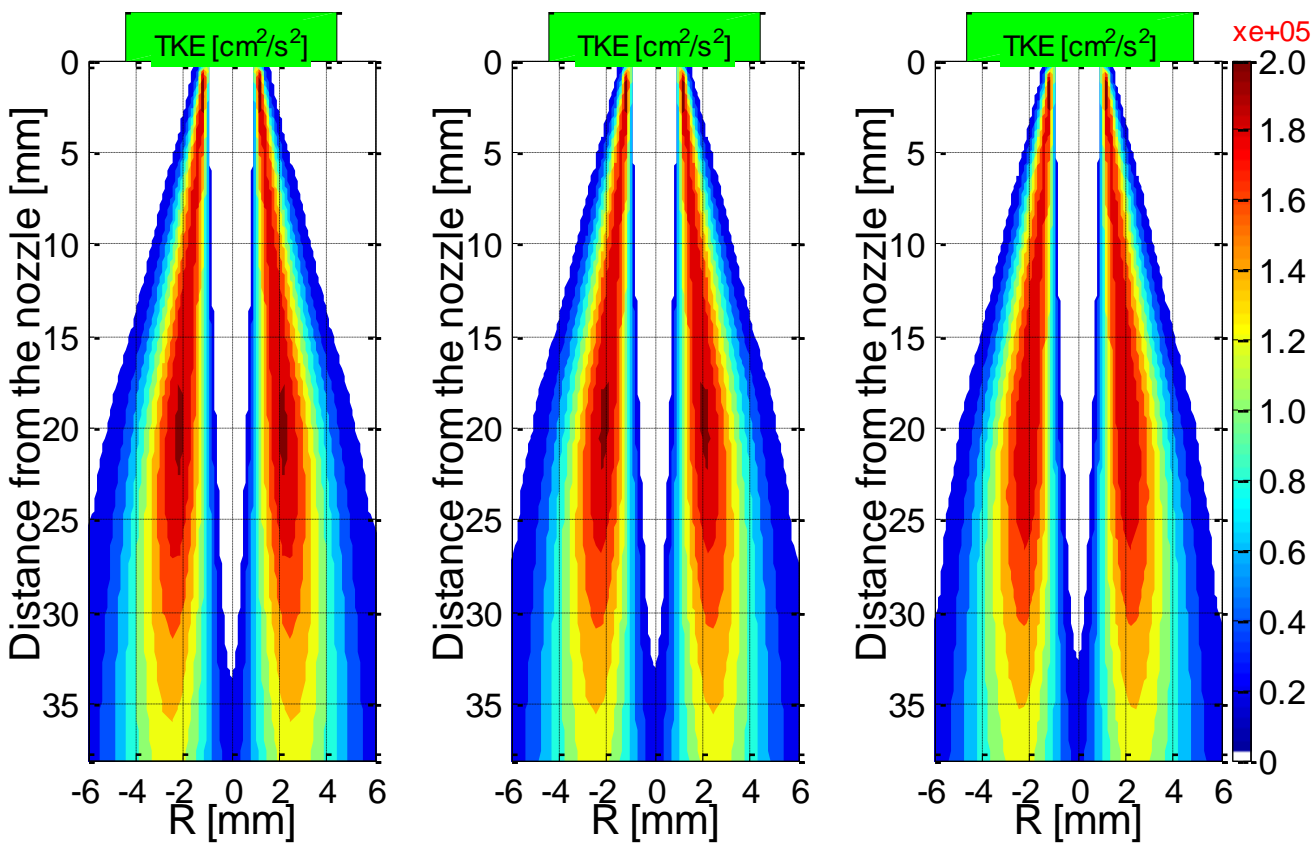

(a)

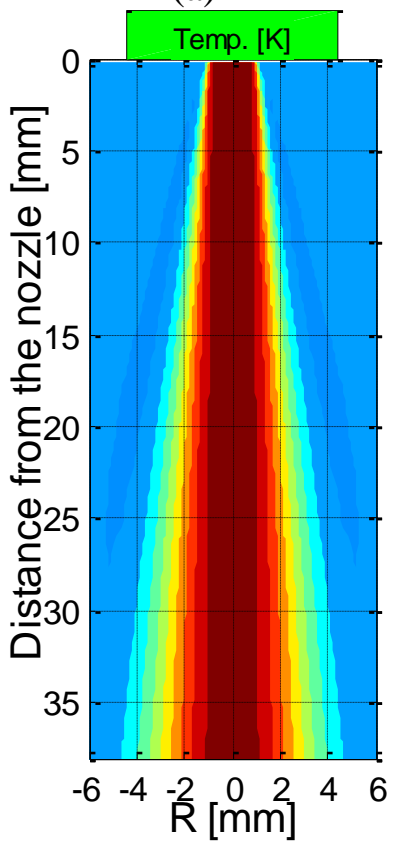

(b)

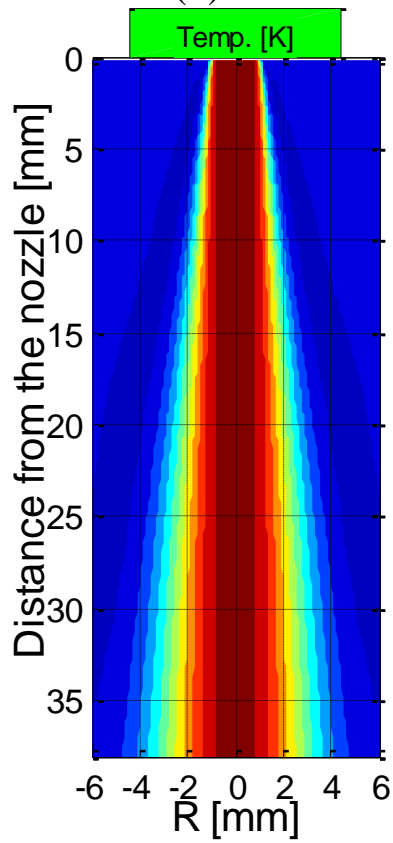

(c)

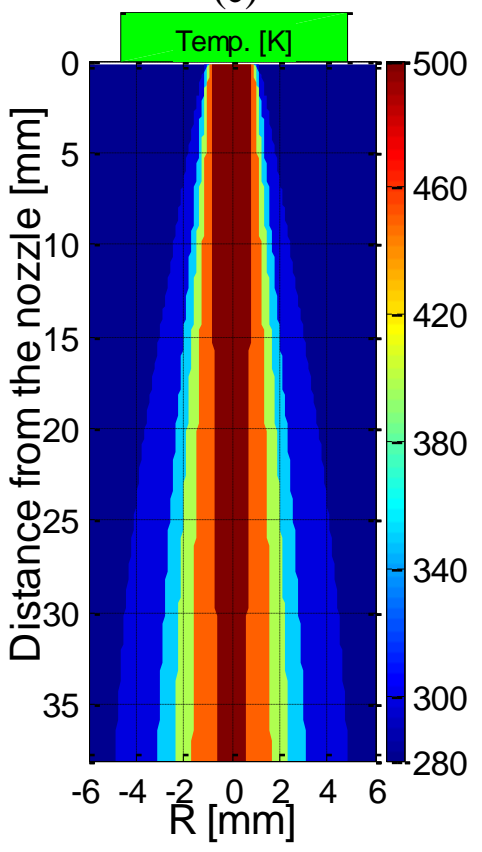

(d)

(e)

(f)

Fig. 19. Turbulent kinetic energy and temperature at 7ms ASI. (a) and (d) Case 9. (b) and (e) Case 1. (c) and (f) Case 10. 


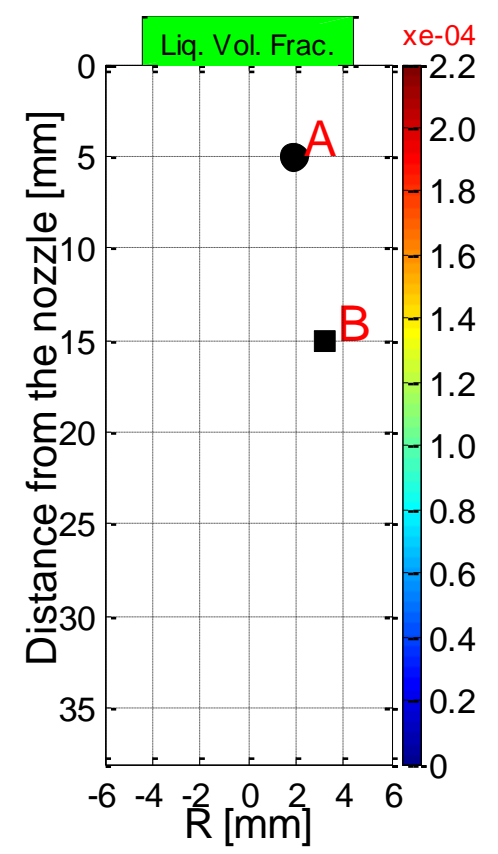

(a)

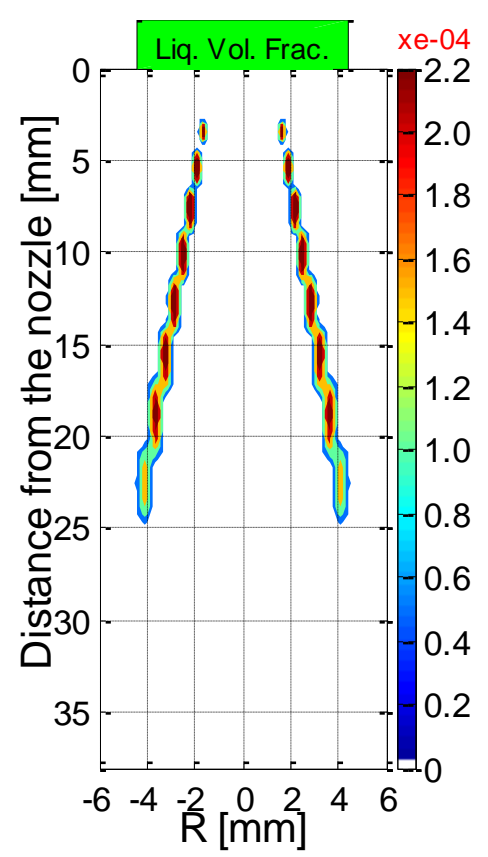

(b)

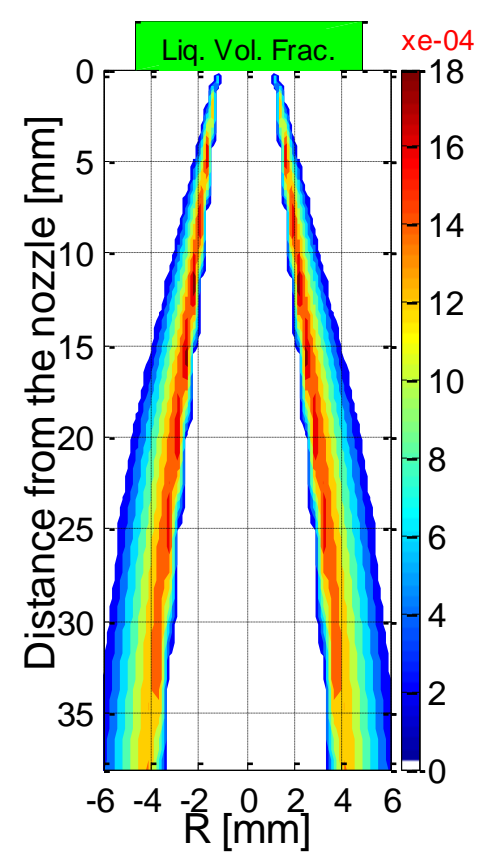

(c)

Fig. 20. Liquid volume fraction at 7ms ASI. (a) Case 9. (b) Case 1. (c) Case 10. Points A and B are shown in (a).

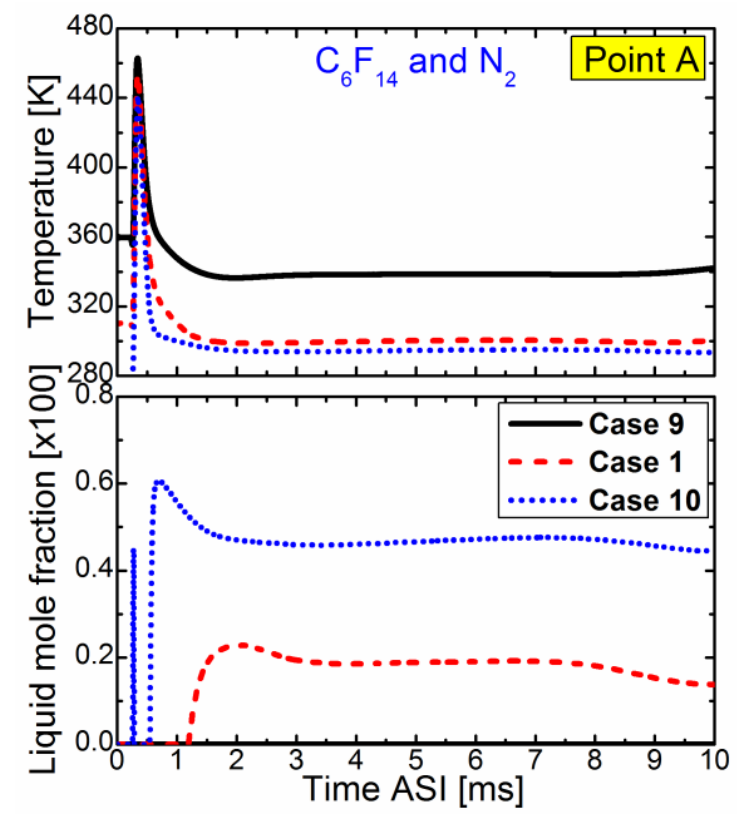

(a)

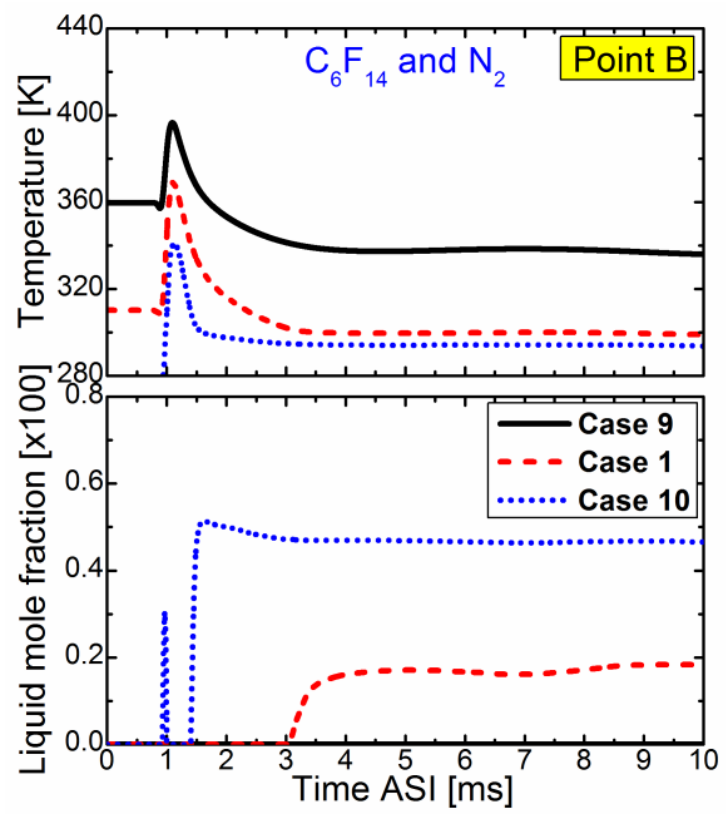

(b)

Fig. 21. Evolution of temperature and liquid mole fraction for Cases 9, 1 and 10. (a) Point A. (b) Point B. 


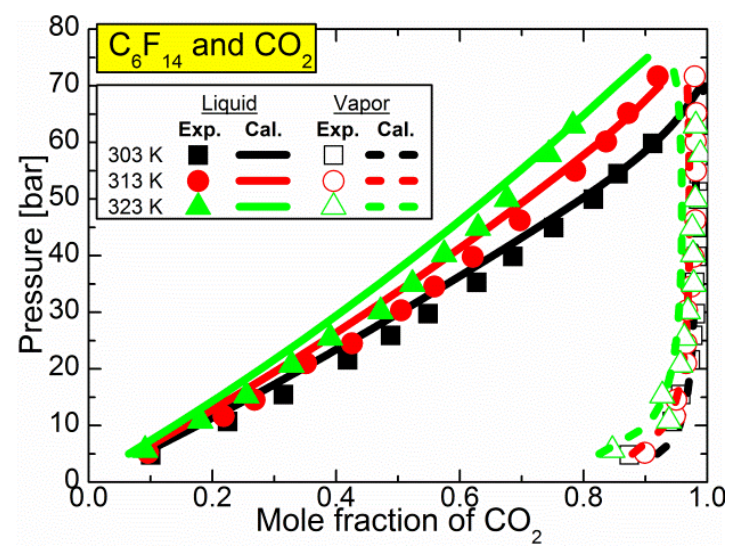

Fig. C1. Vapor-liquid equilibria calculations for binary mixture of $\mathrm{C}_{6} \mathrm{~F}_{14}$ and $\mathrm{CO}_{2}$ compared with experimental data. 